\title{
Microscopic-macroscopic approach for binding energies with the Wigner-Kirkwood method
}

\author{
A. Bhagwat, ${ }^{1, *}$ X. Viñas, ${ }^{2}$ M. Centelles, ${ }^{2}$ P. Schuck, ${ }^{3,4}$ and R. Wyss ${ }^{1}$ \\ ${ }^{1}$ KTH (Royal Institute of Technology), Alba Nova University Center, Department of Nuclear Physics, S-10691 Stockholm, Sweden \\ ${ }^{2}$ Departament d'Estructura i Constituents de la Matèria and Institut de Ciències del Cosmos, Facultat de Física, Universitat de Barcelona, \\ Diagonal 647, E-08028 Barcelona, Spain \\ ${ }^{3}$ Institut de Physique Nucléaire, IN2P3-CNRS, Université Paris-Sud, F-91406 Orsay-Cédex, France \\ ${ }^{4}$ Laboratoire de Physique et Modélisation des Milieux Condensés, CNRS and Université Joseph Fourier, 25 Avenue des Martyrs, \\ Boîte Postale 166, F-38042 Grenoble Cedex 9, France
}

(Received 12 November 2009; revised manuscript received 28 March 2010; published 30 April 2010)

\begin{abstract}
The semiclassical Wigner-Kirkwood $\hbar$ expansion method is used to calculate shell corrections for spherical and deformed nuclei. The expansion is carried out up to fourth order in $\hbar$. A systematic study of Wigner-Kirkwood averaged energies is presented as a function of the deformation degrees of freedom. The shell corrections, along with the pairing energies obtained by using the Lipkin-Nogami scheme, are used in the microscopic-macroscopic approach to calculate binding energies. The macroscopic part is obtained from a liquid drop formula with six adjustable parameters. Considering a set of 367 spherical nuclei, the liquid drop parameters are adjusted to reproduce the experimental binding energies, which yields a root mean square (rms) deviation of $630 \mathrm{keV}$. It is shown that the proposed approach is indeed promising for the prediction of nuclear masses.
\end{abstract}

DOI: 10.1103/PhysRevC.81.044321

PACS number(s): 21.10.Dr, 21.60.-n

\section{INTRODUCTION}

Production and study of loosely bound exotic nuclei using radioactive ion beam facilities is of current interest $[1,2]$. These experiments have given rise to a number of interesting and important discoveries in nuclear physics, like neutron and proton halos, thick skins, disappearance of magicity at the conventional numbers and appearance of new magic numbers, etc. Furthermore, advances in detector systems, and in particular, the development of radioactive beam facilities like Spiral, REX-Isolde, FAIR, and the future FRIB may allow one to investigate new features of atomic nuclei in a novel manner.

The study of nuclear masses and the systematics thereof is of immense importance in nuclear physics. With the advent of mass spectrometry, it is possible to measure masses of some of the short-lived nuclei spanning almost the entire periodic table [3,4]. For example, the isotope separator online (ISOL)based mass analyzer for superheavy atoms (MASHA) [5,6] coming up at JINR-Dubna will be able to directly measure the masses of separated atoms in the range $112 \leqslant Z \leqslant 120$. The limitation on measurements is set by the shortest measurable half-life, $T_{1 / 2} \sim 1.0 \mathrm{~s}$ [5]. The JYFLTRAP [7] developed at the University of Jyväskylä, on the other hand, enables one to measure masses of stable as well as highly neutron-deficient nuclei (for masses up to $A=120$ ) with very high precision $(\sim 50 \mathrm{keV})$ [7].

On the theoretical front as well, considerable progress has already been achieved in the accurate prediction of the nuclear masses, and it is still being pursued vigorously by a number of groups around the globe. This is of great importance because an accurate knowledge of the nuclear masses plays a decisive role in a reliable description of processes like the

\footnotetext{
*ameeya@kth.se; Present address: Dept. of Physics, IIT Gandhinagar, India.
}

astrophysical r-process (see, for example, Ref. [3]). There are primarily two distinct approaches to calculate masses: (a) the microscopic nuclear models based on density functional theory like Skyrme [8,9] and Gogny [10], Hartree-FockBogoliubov, or relativistic mean field (RMF) models [11], and (b) microscopic-macroscopic (Mic-Mac) models [12-15].

The Mic-Mac models are based on the well-known Strutinsky theorem. According to this, the nuclear binding energy, hence the mass, can be written as the sum of a smooth part and an oscillatory part that has its origins in the quantum mechanical shell effects. The latter consists of the shell correction energy and the pairing correlation energy, which in the Mic-Mac models are evaluated in an external potential well. The smooth part is normally taken from the liquid drop models of different degrees of sophistication. The largest uncertainties arise in the calculation of shell corrections. The shell correction is calculated by taking the difference between the total quantum mechanical energy of the given nucleus, and the corresponding "averaged" energy. Usually, the averaging is achieved by the well-established Strutinsky scheme $[16,17]$. This technique of calculating the averaged energies runs into practical difficulties for finite potentials because, for carrying out the Strutinsky averaging, one requires the discrete single-particle spectrum with cutoff well above (at least $3 \hbar \omega_{0}, \hbar \omega_{0}$ being the major shell spacing) the Fermi energy. For a realistic potential, this condition is not met because continuum may start within $\sim \hbar \omega_{0}$ of the Fermi energy. Standard practice is to discretize the continuum by diagonalizing the Hamiltonian in a basis of optimum size. A number of Mic-Mac calculations with varying degree of success are available in the literature (see, e.g., Refs. [12-15]). The Mic-Mac models typically yield better than $\sim 0.7-\mathrm{MeV}$ rms deviation in the masses. All these models agree reasonably well with each other and with experiment, but deviate widely among themselves in the regions far away from the valley of stability. 
The semiclassical Wigner-Kirkwood (WK) approach [18-25], on the other hand, makes no explicit reference to the single-particle spectrum, and achieves an accurate averaging of the given one-body Hamiltonian. Thus, the WK approach is a good alternative to the conventional Strutinsky smoothing scheme. The quantum mechanical energy is calculated by diagonalizing the one-body Hamiltonian in the axially symmetric deformed harmonic oscillator basis with 15 shells. More details about this quantal calculation are given in Appendix A. The difference between the total quantum mechanical energy and the WK energy in the external potential well yields the value of the shell correction for a given system. In the present work, we propose to carry out a reliable microscopic-macroscopic calculation of the nuclear binding energies (and hence the masses), employing the semiclassical Wigner-Kirkwood (WK) $\hbar$ expansion [18-25] for the calculation of shell corrections instead of the Strutinsky scheme. We are aware that the finite size of the harmonic oscillator basis induces some uncertainty in the quantal energies that in this case cannot be compensated by the WK semiclassical energy. We will discuss explicitly this problem in Appendix A. An exploratory study of using the WK method to compute the smooth part of the energy was reported earlier to test the validity of the Strutinsky scheme, especially near the drip lines [26].

It is known that the WK level density $\left(g_{\mathrm{WK}}(\varepsilon)\right)$ with the $\hbar^{2}$ correction term exhibits a $\varepsilon^{-1 / 2}$ divergence as $\varepsilon \rightarrow 0$ for potentials that vanish at large distances as, for instance, Woods-Saxon potentials (see, e.g., Ref. [27]). The Strutinsky level density, on the contrary, exhibits only a prominent peak as $\varepsilon \rightarrow 0$. It was therefore concluded in Ref. [28] that the divergence of the WK level density as $\varepsilon \rightarrow 0$ is unphysical, and the Strutinsky smoothed level density should be preferred. It should, however, be noted that the WK level densities, energy densities, etc., have to be understood in the mathematical sense of distributions and, consequently, only integrated quantities are meaningful. In fact, it was shown [25] that the integrated quantities such as the accumulated level densities are perfectly well behaved, even for $\varepsilon \rightarrow 0$.

Pairing correlations are important for open shell nuclei. In the present work, these are taken into account in the approximate particle number projected Lipkin-Nogami scheme [29-31]. Odd-even and odd-odd nuclei are treated in an entirely microscopic fashion (odd nucleon blocking method in the uniform filling approximation), allowing an improved determination of odd-even mass differences (see, e.g., the discussion in Ref. [32]). The majority of nuclei in the nuclear chart are deformed. In particular, it is well known that inclusion of deformation is important for reliable predictions of nuclear masses. Therefore, here we incorporate in all three deformation degrees of freedom $\left(\beta_{2}, \beta_{4}, \gamma\right)$. To our knowledge, no such detailed and extensive calculation based on the WK method is available in the literature.

The paper is organized as follows. We review the WK expansion in Sec. II. The choice of the nuclear, spin-orbit, and Coulomb potentials forms the subject matter of Sec. III. Details of the WK calculations are discussed in Sec. IV. A systematic study of the WK energies for neutrons and protons as a function of the deformation degrees of freedom is presented in Sec. V. The shell corrections for the chains of the $\mathrm{Gd}$, $\mathrm{Dy}$, and $\mathrm{Pb}$ isotopes obtained by using our formalism are reported and compared with those calculated employing the traditional Strutinsky averaging technique in Sec. VI. Sec. VII contains a brief discussion on the Lipkin-Nogami pairing scheme. As an illustrative example, the calculation of the binding energies for selected 367 spherical nuclei is presented and discussed in Sec. VIII. Sec. IX contains our summary and future outlook. Details about the quantum mechanical calculation of the energy of a set of nucleons submitted to an external Woods-Saxon potential as well as the effect of the finite size of the deformed harmonic oscillator basis are given in Appendix A. Supplementary material can be found in Appendices B and C.

\section{SEMICLASSICAL WIGNER-KIRKWOOD EXPANSION}

Following Ref. [20], we consider a system of $N$ noninteracting fermions at zero temperature. Suppose that these fermions are moving in a given one-body potential including the spin-orbit interaction. To determine the smooth part of the energy of such a system, we start with the quantal partition function for the system,

$$
Z(\beta)=\operatorname{Tr}(\exp (-\beta \hat{H})) .
$$

Here, $\hat{H}$ is the Hamiltonian of the system, given by

$$
\hat{H}=\frac{-\hbar^{2}}{2 m} \nabla^{2}+V(\vec{r})+\hat{V}_{L S}(\vec{r}),
$$

where $V(\vec{r})$ is the one-body central potential and $\hat{V}_{L S}(\vec{r})$ is the spin-orbit interaction.

To average out shell effects, the simplest one could do is replace the partition function in the above expression by the classical partition function; that is, one replaces the Hamiltonian in Eq. (1) by the corresponding classical Hamiltonian. This yields the well-known Thomas-Fermi equations for particle number and energy. Way back in the 1930s, Wigner [18] and Kirkwood [19] developed a systematic expansion of the partition function in powers of the Planck's constant, $\hbar$, its first term being the classical partition function. Details of this method can be found in Refs. [18-23]. Such expansion of the quantal partition function in powers of $\hbar$ is often known as the Wigner-Kirkwood expansion. Systematic corrections to the Thomas-Fermi energy can be obtained by using the WK expansion.

In this work, we shall use the WK expansion up to the fourth order. For brevity, we represent the potentials and form factors without mentioning the dependence on the position vector. Ignoring the spin-orbit interaction, the WK expansion of the partition function, correct up to the fourth order is given [20]:

$$
\begin{aligned}
Z^{(4)}(\beta)= & \frac{\beta^{-3 / 2}}{4 \pi^{3 / 2}}\left(\frac{2 m}{\hbar^{2}}\right)^{3 / 2} \int d \vec{r} e^{-\beta V}\left\{1-\frac{\beta^{2} \hbar^{2}}{24 m} \nabla^{2} V\right. \\
& +\frac{\beta^{3}}{1440}\left(\frac{\hbar^{2}}{2 m}\right)^{2}\left[-7 \nabla^{4} V+5 \beta\left(\nabla^{2} V\right)^{2}\right. \\
& \left.\left.+\beta \nabla^{2}(\nabla V)^{2}\right]\right\} .
\end{aligned}
$$


The spin-orbit interaction, in general, can be written as

$$
\hat{V}_{L S}=\frac{\iota \kappa \hbar^{2}}{2 m}(\vec{\nabla} f \times \vec{\nabla}) \cdot \hat{\sigma}
$$

where $\hat{\sigma}$ is the unit Pauli matrix, $\kappa$ is the strength of spinorbit interaction, and $f$ is the spin-orbit form factor. With the inclusion of such spin-orbit interaction, the WK expansion for the full partition function splits up into two parts:

$$
Z_{\mathrm{WK}}^{(4)}(\beta)=Z^{(4)}(\beta)+Z_{L S}^{(4)}(\beta) .
$$

Here, $Z^{(4)}(\beta)$ is given by Eq. (3), and the spin-orbit contribution to the partition function, correct up to the fourth order in $\hbar$, reads [20]

$$
\begin{aligned}
Z_{L S}^{(4)}= & \frac{\kappa^{2} \beta^{1 / 2}}{8 \pi^{3 / 2}}\left(\frac{2 m}{\hbar^{2}}\right)^{1 / 2} \int d \vec{r} e^{-\beta V}(\nabla f)^{2}+\frac{\beta^{1 / 2}}{96 \pi^{3 / 2}} \\
& \times\left(\frac{\hbar^{2}}{2 m}\right)^{1 / 2} \int d \vec{r} e^{-\beta V}\left\{\kappa^{2} f_{2}-2 \kappa^{3} f_{3}+2 \kappa^{4} f_{4}\right\},
\end{aligned}
$$

where

$$
\begin{aligned}
f_{2}= & -\beta(\nabla f)^{2}\left(\nabla^{2} V\right)+\frac{1}{2} \nabla^{2}(\nabla f)^{2} \\
& -\left(\nabla^{2} f\right)^{2}+\nabla f \cdot \nabla\left(\nabla^{2} f\right) \\
f_{3}= & (\nabla f)^{2} \nabla^{2} f-\frac{1}{2} \nabla f \cdot \nabla(\nabla f)^{2} \\
f_{4}= & (\nabla f)^{4} .
\end{aligned}
$$

The level density $g_{\mathrm{WK}}$, particle number $N$, and energy $E$ can be calculated directly from the WK partition function by Laplace inversion:

$$
\begin{aligned}
g_{\mathrm{WK}}(\epsilon) & =\mathcal{L}_{\epsilon}^{-1} Z_{\mathrm{WK}}^{(4)}(\beta), \\
N & =\mathcal{L}_{\lambda}^{-1}\left(\frac{Z_{\mathrm{WK}}^{(4)}(\beta)}{\beta}\right),
\end{aligned}
$$

and

$$
E=\lambda N-\mathcal{L}_{\lambda}^{-1}\left(\frac{Z_{\mathrm{WK}}^{(4)}(\beta)}{\beta^{2}}\right)
$$

where $\lambda$ is the chemical potential, fixed by demanding the right particle number, and $\mathcal{L}_{\lambda(\epsilon)}^{-1}$ denotes the Laplace inversion. Using the identity,

$\mathcal{L}_{\lambda}^{-1}\left(\frac{e^{-\beta V}}{\beta^{\mu}}\right)=\frac{(\lambda-V)^{\mu-1}}{\Gamma(\mu)} \Theta(\lambda-V), \quad$ for $\quad \mu>0$,

and noting that to get inverse Laplace transforms in convergent form,

$$
e^{-\beta V}=\frac{-1}{\beta} \frac{\partial e^{-\beta V}}{\partial V}
$$

one obtains the level density for each kind of nucleons assuming spin degeneracy:

$$
\begin{aligned}
g_{\mathrm{WK}}(\epsilon)= & \frac{1}{3 \pi^{2}}\left(\frac{2 m}{\hbar^{2}}\right)^{3 / 2} \int d \vec{r}\left\{\frac{3}{2}(\epsilon-V)^{1 / 2}\right. \\
& +\frac{\hbar^{2}}{4 m}\left[\frac{3}{4} \kappa^{2}(\nabla f)^{2}(\epsilon-V)^{-1 / 2}\right. \\
& \left.\left.+\frac{1}{16} \Delta V(\epsilon-V)^{-3 / 2}\right]\right\} \Theta(\epsilon-V),
\end{aligned}
$$

the particle number:

$$
\begin{aligned}
N= & \frac{1}{3 \pi^{2}}\left(\frac{2 m}{\hbar^{2}}\right)^{3 / 2} \int d \vec{r}\left[(\lambda-V)^{3 / 2}-\frac{\hbar^{2}}{32 m}(\lambda-V)^{-1 / 2}\right. \\
& \left.\times \nabla^{2} V+\frac{3 \hbar^{2} \kappa^{2}}{8 m}(\lambda-V)^{1 / 2}(\nabla f)^{2}\right] \Theta(\lambda-V),
\end{aligned}
$$

and the energy:

$$
\begin{aligned}
E= & \lambda N-\frac{1}{3 \pi^{2}}\left(\frac{2 m}{\hbar^{2}}\right)^{3 / 2} \\
& \times \int d \vec{r}\left\{\frac{2}{5}(\lambda-V)^{5 / 2}-\frac{\hbar^{2}}{16 m}(\lambda-V)^{1 / 2} \nabla^{2} V\right\} \Theta(\lambda-V) \\
& -\frac{1}{5760 \pi^{2}}\left(\frac{\hbar^{2}}{2 m}\right)^{1 / 2}\left\{\int d \vec{r}(\lambda-V)^{-1 / 2}\left[-7 \nabla^{4} V\right]\right. \\
& \left.-\frac{1}{2} \int d \vec{r}(\lambda-V)^{-3 / 2}\left[5\left(\nabla^{2} V\right)^{2}+\nabla^{2}(\nabla V)^{2}\right]\right\} \Theta(\lambda-V) \\
& -\frac{\kappa^{2}}{6 \pi^{2}}\left(\frac{2 m}{\hbar^{2}}\right)^{1 / 2} \int d \vec{r}(\lambda-V)^{3 / 2}(\nabla f)^{2} \Theta(\lambda-V) \\
& -\frac{1}{48 \pi^{2}}\left(\frac{\hbar^{2}}{2 m}\right)^{1 / 2} \int d \vec{r}(\lambda-V)^{1 / 2}\left\{\kappa ^ { 2 } \left[\frac{1}{2} \nabla^{2}(\nabla f)^{2}\right.\right. \\
& \left.-\left(\nabla^{2} f\right)^{2}+\nabla f \cdot \nabla\left(\nabla^{2} f\right)-\frac{(\nabla f)^{2} \nabla^{2} V}{2(\lambda-V)}\right] \\
& -2 \kappa^{3}\left[(\nabla f)^{2} \nabla^{2} f-\frac{1}{2} \nabla f \cdot \nabla(\nabla f)^{2}\right] \\
& \left.+2 \kappa^{4}(\nabla f)^{4}\right\} \Theta(\lambda-V) .
\end{aligned}
$$

It should be noted that we have explicitly assumed that all the derivatives of the potential $V$ and the spin-orbit form factor $f$ exist. The expansion defined here is therefore not valid for potentials with sharp surfaces. This automatically puts a restriction on the choice of the Coulomb potential: The conventional uniform distribution approximation for the charge distribution cannot be used in the present case. We shall discuss this point at greater length in the next section. The integrals in the above expressions are cut off at the turning points, defined via the step function. The chemical potential $\lambda$ appearing in these equations is determined from Eq. (16), separately for neutrons and protons. Furthermore, it is interesting to note that the spin-orbit contribution to the particle number $N$ as well as to the energy $E$ appears only in the second order in $\hbar$. Secondly, the level density and particle number are 
calculated only up to the order $\hbar^{2}$. It can be shown [20] that for the expansion correct up to the fourth order in $\hbar$, it is sufficient to take $Z_{\mathrm{WK}}^{(4)}$ up to order $\hbar^{2}$ in Eq. (11) to find the chemical potential (and hence the particle number), whereas one has to take the full partition function $Z_{\mathrm{WK}}^{(4)}$ up to order $\hbar^{4}$ in Eq. (12) to compute the energy in the WK approach.

The divergent terms appearing in Eq. (17) are treated by differentiation with respect to the chemical potential. Explicitly,

$$
\begin{aligned}
& \partial_{\lambda}(\lambda-V)^{1 / 2}=\frac{1}{2}(\lambda-V)^{-1 / 2} \\
& \partial_{\lambda}^{2}(\lambda-V)^{1 / 2}=-\frac{1}{4}(\lambda-V)^{-3 / 2} .
\end{aligned}
$$

In practice, the differentiation with respect to chemical potential is carried out after evaluation of the relevant integrals. Numerically, this approach is found to be stable. Its reliability has been checked explicitly by reproducing the values of fourth-order WK corrections quoted in Ref. [20].

The WK expansion thus defined, converges very rapidly for the harmonic oscillator potential: The second-order expansion itself is enough for most practical purposes. The convergence for the Woods-Saxon potential, however, is slower than that for the harmonic oscillator potential, but it is adequate [33]. For example, for $\sim 126$ particles, the Thomas-Fermi energy is typically of the order of $10^{3} \mathrm{MeV}$, the second-order $\left(\hbar^{2}\right)$ correction contributes a few tens of $\mathrm{MeVs}$, and the fourth-order $\left(\hbar^{4}\right)$ correction yields a contribution of the order of $1 \mathrm{MeV}$. This point will be discussed in greater details later. It is also important to note that the $\mathrm{WK} \hbar$ expansion of the density matrix has a variational character and that a variational theory based on a strict expansion in the powers of $\hbar$ was established [34].

The WK approach presented here should be distinguished from the extended Thomas-Fermi (ETF) approach. Divergence problems at the classical turning points (see the particle number and energy expressions above) can be eliminated by expressing the kinetic energy density as a functional of the local density. This is achieved by eliminating the chemical potential, the local potential, and the derivatives of the local potential (for further details, see Ref. [35]). It cannot be accomplished in closed form and has to be done iteratively, leading to a functional series for the kinetic energy density. The resulting model is what is often referred to as the ETF approach. The WK approach as presented here, in this sense, is the starting point for the ETF approach (further details of ETF can be found in Refs. [22,23,25,36-38]). The conventional ETF approach exhibits somewhat slower convergence properties, which has been attributed to a nonoptimal sorting out of terms of each given power in $\hbar[25,35]$.

\section{CHOICE OF POTENTIAL}

\section{A. Form of the nuclear potential}

The spherically symmetric nuclear mean field is well represented by the Woods-Saxon (WS) form [39], given by

$$
V(r)=\frac{V_{0}}{1+\exp \left[\left(r-R_{0}\right) / a\right]},
$$

where $V_{0}$ is the strength of the potential, $R_{0}$ is the halfdensity radius, and $a$ is the diffuseness parameter. The WS form factor defined here, can be easily generalized to take the deformation effects into account. Note that the distance function $l(r)=r-R_{0}$ appearing in Eq. (20) can be interpreted as the minimum distance of a given point to the nuclear surface, defined by $r=R_{0}$. One might thus generalize it to the case of deformed surfaces as well. Using the standard expansion in terms of spherical harmonics, a general deformed surface may be defined by the relation $r=r_{s}$, where

$$
r_{s}=C R_{0}\left(1+\sum_{\lambda, \mu} \alpha_{\lambda, \mu} Y_{\lambda, \mu}\right) .
$$

Here, the $Y_{\lambda, \mu}$ functions are the usual spherical harmonics and the constant $C$ is the volume conservation factor (the volume enclosed by the deformed surface should be equal to the volume enclosed by an equivalent spherical surface of radius $R_{0}$ ):

$$
C=\left\{\frac{1}{4 \pi} \int_{\Omega}\left[1+\sum_{\lambda, \mu} \alpha_{\lambda, \mu} Y_{\lambda, \mu}(\Omega)\right]^{3} d \Omega\right\}^{-1 / 3} .
$$

The distance function to be used in the WS potential would be the minimum distance of a given point to the nuclear surface defined by $r=r_{s}$. Such definition has been used quite extensively in the literature, with good success (see, e.g., Refs. [40-44]). However, in the present case, this definition is not convenient because it should be noted that the calculation of this distance function involves the minimization of a segment from the given point to the nuclear surface. This, in turn, implies that each calculation of the distance function (for given $r, \theta$, and $\phi$ coordinates we are assuming a spherical polar coordinate system here) involves the calculation of two surface angles $\theta_{s}$ and $\phi_{s}$, and these are implicit functions of $r$, $\theta$, and $\phi$ (see Fig. 8 in Appendix B for details). Because the WK calculations involve differentiation of the WS function, one also needs to differentiate $\theta_{s}$ and $\phi_{s}$, which are implicit functions of $r, \theta$, and $\phi$.

Alternatively, the distance function for the deformed Woods-Saxon potential can be written down by demanding that the rate of change of the potential calculated normal to the nuclear surface and evaluated at the nuclear surface should be a constant [45], which, indeed, is the case for the spherical Woods-Saxon form factor. Thus,

$$
\{\hat{n} \cdot \nabla V(\vec{r})\}_{r=r_{s}}=\text { constant, }
$$

where $\hat{n}$ is the unit vector normal to the surface $\left(r=r_{s}\right)$ and is given by

$$
\hat{n}=\frac{\nabla\left(r-r_{s}\right)}{\left|\nabla\left(r-r_{s}\right)\right|} .
$$

In fact, the above condition [Eq. (23)] is related to the observation that the second derivative of the spherical WoodsSaxon form factor vanishes at the nuclear surface, defined by $r=R_{0}$. The resulting distance function is given [46]:

$$
l(\vec{r})=\frac{r-r_{s}}{\left|\nabla\left(r-r_{s}\right)\right|_{r=r_{s}}},
$$


where $r_{s}$ is as defined in Eq. (21). The denominator is evaluated at $r=r_{s}$. Writing the $\theta$ and $\phi$ derivatives of $r_{s}$ as $A$ and $B$, respectively, we get

$$
l(\vec{r})=\frac{\left(r-r_{s}\right)}{\sqrt{1+\gamma^{2} / r_{s}^{2}}},
$$

with

$$
\gamma^{2}=A^{2}+B^{2} \csc ^{2} \theta
$$

In the present work, we use the distance function as defined in Eq. (25). The WS potential thus reads

$$
V(\vec{r})=\frac{V_{0}}{1+\exp [l(\vec{r}) / a]} .
$$

It is straightforward to check that the Woods-Saxon potential defined with the distance function as given by Eq. (25) satisfies the condition Eq. (23). Substituting this Woods-Saxon potential in $\hat{n} \cdot \nabla V(\vec{r})$, we get

$$
\begin{aligned}
\hat{n} \cdot \nabla V(\vec{r})= & \frac{V_{0}}{a} f(\vec{r})[f(\vec{r})-1] \hat{n} \cdot \nabla l(\vec{r}) \\
= & \frac{V_{0}}{a} f(\vec{r})[f(\vec{r})-1]\left[\frac{\left|\nabla\left(r-r_{s}\right)\right|}{\left|\nabla\left(r-r_{s}\right)\right|_{r=r_{s}}}\right. \\
& \left.+\left(r-r_{s}\right) \frac{\nabla\left(r-r_{s}\right)}{\left|\nabla\left(r-r_{s}\right)\right|} \cdot \nabla \frac{1}{\left|\nabla\left(r-r_{s}\right)\right|_{r=r_{s}}}\right] .
\end{aligned}
$$

Here, $f(\vec{r})=\{1+\exp [l(\vec{r}) / a]\}^{-1}$ is the Woods-Saxon form factor. Clearly, at the surface defined by $r=r_{s}$, the quantity $\hat{n} \cdot \nabla V(\vec{r})$ is constant.

\section{B. Deformation parameters}

In practice, we consider three deformation degrees of freedom, namely, $\beta_{2}, \beta_{4}$, and $\gamma$. These parameters are related with the parameters $\alpha_{\lambda, \mu}$ introduced in Eq. (21). Note that for the given choice of deformation parameters, $\lambda$ takes values 2 and 4 . The projection $\mu$ takes the values 0 and \pm 2 for $\lambda=2$ and the values $0, \pm 2$, and \pm 4 for $\lambda=4$. Furthermore, the existence of symmetry planes $(x, y),(y, z)$, and $(z, x)$ implies that [42]

$$
\alpha_{2,2}=\alpha_{2,-2}, \quad \alpha_{4,2}=\alpha_{4,-2}, \quad \alpha_{4,4}=\alpha_{4,-4}
$$

Thus, we get

$$
\begin{aligned}
r_{s}(\theta, \phi)= & C R_{0}\left\{1+\alpha_{2,0} Y_{2,0}(\theta)\right. \\
& +\alpha_{2,2}\left[Y_{2,2}(\theta, \phi)+Y_{2,-2}(\theta, \phi)\right]+\alpha_{4,0} Y_{4,0}(\theta) \\
& +\alpha_{4,2}\left[Y_{4,2}(\theta, \phi)+Y_{4,-2}(\theta, \phi)\right] \\
& \left.+\alpha_{4,4}\left[Y_{4,4}(\theta, \phi)+Y_{4,-4}(\theta, \phi)\right]\right\}
\end{aligned}
$$

where

$$
\begin{aligned}
& \alpha_{2,0}=\beta_{2} \cos \gamma \\
& \alpha_{2,2}=-\sqrt{\frac{1}{2}} \beta_{2} \sin \gamma \\
& \alpha_{4,0}=\frac{1}{6} \beta_{4}\left(5 \cos ^{2} \gamma+1\right)
\end{aligned}
$$

$$
\begin{aligned}
\alpha_{4,2} & =-\sqrt{\frac{30}{144}} \beta_{4} \sin 2 \gamma \\
\alpha_{4,4} & =\sqrt{\frac{70}{144}} \beta_{4} \sin ^{2} \gamma .
\end{aligned}
$$

For further details, see Ref. [42].

\section{Woods-Saxon parameters}

The parameters [47] appearing in the Woods-Saxon potential are as defined below:

(i) Central potential

(a) Strength:

$$
V_{0}=-U_{0}\left\{1 \mp U_{1} \frac{N-Z}{A}\right\},
$$

with $U_{0}=53.754 \mathrm{MeV}$ and $U_{1}=0.791$.

(b) Half-density radius:

$$
R_{0}=r_{0} A^{1 / 3}\left\{1 \mp c_{1} \frac{N-Z}{A}\right\}+c_{2},
$$

with $r_{0}=1.19 \mathrm{fm}, c_{1}=-0.116$, and $c_{2}=0.235$ fm.

(c) Diffuseness parameter: This is assumed to be the same for neutrons and protons, and has the value $a=0.637 \mathrm{fm}$.

(ii) Spin-orbit potential

(a) Strength:

$$
V_{\mathrm{SO}}=\lambda_{0} U_{0} \frac{\hbar^{2}}{4 m^{2}}\left\{1 \mp U_{2} \frac{N-Z}{A}\right\},
$$

with $U_{0}=53.754 \mathrm{MeV}, \lambda_{0}=29.494$, and $U_{2}=$ 0.162 .

(b) Half-density radius and diffuseness parameter:

These are taken to be the same as those for the central potential.

In these expressions, the $+(-)$ sign holds for protons (neutrons).

The parameters have the isospin dependence of the central and spin-orbit potentials "built-in." This potential yields a reasonably good description of charge radii (both magnitude and isospin dependence) as well as of moments of inertia for a wide range of nuclei. It was used extensively in the total Routhian surface (TRS) calculations, and it was quite successful in accurately reproducing energies of single-particle as well as collective states [48].

\section{Coulomb potential}

The Coulomb potential is calculated by folding the point proton density distribution $\rho\left(\vec{r}^{\prime}\right)$, assumed to be of WoodsSaxon form. For simplicity, its parameters are assumed to be the same as those for the nuclear potential of protons. The reason for using folded potential here is, as we have indicated in Sec. II, the WK expansion is not valid for potentials with sharp surfaces. 


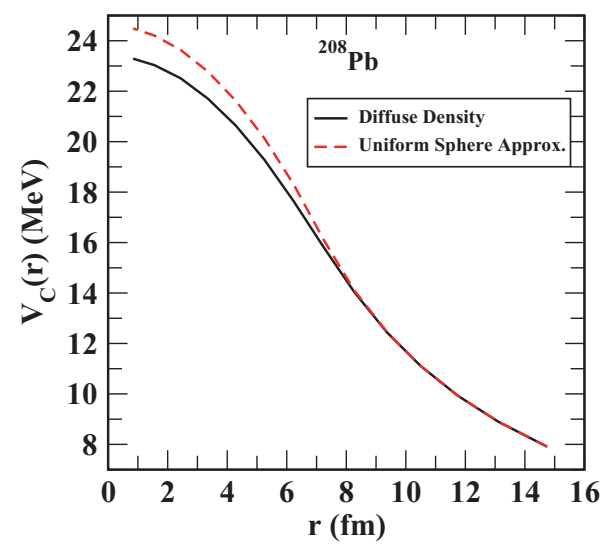

FIG. 1. (Color online) Coulomb potentials obtained by using diffuse density and sharp surface approximation for ${ }^{208} \mathrm{~Pb}$.

The Coulomb potential for the extended charge distribution is given:

$$
V_{C}(\vec{r})=e^{2} \int \rho\left(\vec{r}^{\prime}\right) \frac{1}{\left|\vec{r}-\vec{r}^{\prime}\right|} d \vec{r}^{\prime} .
$$

Here,

$$
\left|\vec{r}-\vec{r}^{\prime}\right|=\left\{r^{2}+r^{\prime 2}-2 r r^{\prime} \cos \Psi\right\}^{1 / 2}
$$

where

$$
\cos \Psi=\cos \theta \cos \theta^{\prime}+\sin \theta \sin \theta^{\prime} \cos \left(\phi-\phi^{\prime}\right),
$$

as explained in Appendix B.

It is instructive at this point, to compare the Coulomb potential calculated from the diffuse density with the corresponding potential obtained by using the conventional uniform density (sharp surface) approximation. Such comparison for ${ }^{208} \mathrm{~Pb}$ is plotted in Fig. 1. The radius parameter for the diffuse density approach as well as for the sharp surface approximation is assumed to be equal to $7.11 \mathrm{fm}$ (see the discussion on the choice of the Woods-Saxon parameters in Sec. III). It can be seen that in the exterior region, the two potentials agree almost exactly, as expected. In the interior, however, the potential obtained from the diffuse density turns out to be somewhat less repulsive than that from the density with sharp surface.

\section{DETAILS OF THE WK CALCULATIONS}

In the present work, we restrict our calculations to three deformation degrees of freedom, namely, $\beta_{2}, \beta_{4}$, and the angle $\gamma$. The inclusion of $\gamma$ allows one to incorporate triaxiality. Thus, the present $\mathrm{WK}$ calculation is genuinely three-dimensional. In principle, it is natural to use a cylindrical coordinate system here. The spherical polar coordinates, however, turn out to be more convenient. The reason is that the cylindrical coordinates involve two length variables and one angular coordinate, which means that the turning points have to be evaluated for two coordinates ( $\rho$ and $z$ ). This makes the calculations very complicated. On the other hand, the spherical polar coordinates involve only one length variable, and thus the turning points are to be evaluated only for one coordinate $(r)$. The numerical integrals involved are evaluated using Gaussian quadrature.

The first step in the WK calculations is the determination of the chemical potential. This has to be done iteratively, using Eq. (16). Since the turning points are determined by the chemical potential, they have to be calculated using a suitable numerical technique at each step. Once the values of the chemical potential are known, the WK energies up to the second order can be calculated in a straightforward way. The fourth-order calculations are very complicated because they require higher-order derivatives of nuclear potentials, spin-orbit form factors, and the Coulomb potential. The former can be evaluated analytically in the present case. The expressions are extremely lengthy, and we do not present them here. Comparatively, the derivatives of the Coulomb potential look simple; the Laplacian and Laplacian of Laplacian are completely straightforward: the former is proportional to the proton density and the latter is just the Laplacian of the WS form factor. However, the calculations also need terms like Laplacian of the gradient squared of the total potential. In the case of protons, this involves one crossed term:

$$
\nabla^{2}\left[\nabla V_{C}(\vec{r}) \cdot \nabla V_{N}(\vec{r})\right]
$$

where $V_{C}$ is the Coulomb potential and $V_{N}$ is the nuclear potential. The determination of such objects is tricky. It turns out that if one uses the form of the Coulomb potential defined above, the calculation of expression Eq. (42) becomes numerically unstable.

There exists an alternative for the Coulomb potential:

$$
V_{C}(\vec{r})=\frac{e^{2}}{2} \int d \vec{r}^{\prime}\left|\vec{r}-\vec{r}^{\prime}\right| \nabla_{\vec{r}^{\prime}}^{2} \rho\left(\vec{r}^{\prime}\right),
$$

where the notation $\nabla_{\vec{r}^{\prime}}^{2}$ means that the Laplacian is calculated with respect to the variables $r^{\prime}, \theta^{\prime}$, and $\phi^{\prime}$. Equations (39) and (43) are exactly equivalent. This is proved explicitly in Appendix C. With this form, one can calculate the first and second derivatives (not the Laplacian) of the Coulomb potential. Calculation of the higher-order derivatives of the Coulomb potential, even with the form defined in Eq. (43), turns out to be numerically unstable. For this purpose, we employ the Poisson's equation. The details are presented in Appendix C. Once all the derivatives are known, the fourth-order WK calculations can be carried out.

It turns out that the WK calculations for protons are very time-consuming. This is due to the fact that the calculation of Coulomb potential [Eq. (39)], in general, involves evaluation of the three-dimensional integral for each point $(r, \theta, \phi)$. Typically, it takes a few tens of minutes to complete one such calculation. This is certainly not desirable because our aim is to calculate the masses of the nuclei spanning the entire periodic table. To speed up the calculations, we use the well-known technique of interpolation. Because we are using spherical polar coordinates, the turning points are to be evaluated only for the radial coordinate, $r$. For the entire WK calculation, the $\theta$ and $\phi$ mesh points remain the same (over the domains 
$[0, \pi]$ and $[0,2 \pi]$, respectively), whereas the $r$ mesh points change from step to step. This happens in particular during the evaluation of the chemical potential. Once the convergence of the particle number equation [Eq. (16)] is achieved, the $r$ mesh points remain fixed as well.

Motivated by the above observations, we apply the following procedure:

(i) Before entering into the actual WK calculations (determination of chemical potential, etc.), for each pair of $\theta$ and $\phi$ mesh points, we calculate the Coulomb potential [Eq. (39)] over a range of $0-16 \mathrm{fm}$ at equidistant radial mesh points (the typical mesh size being $0.1 \mathrm{fm}$ ).

(ii) Next, for each pair of $\theta$ and $\phi$ mesh points, we fit a polynomial of degree 9 in the radial coordinate $r$ to the Coulomb potential calculated in the above step. Thus, the fitting procedure is to be repeated $N_{\theta} \times N_{\phi}$ times, $N_{\theta}\left(N_{\phi}\right)$ being the total number of mesh points for the $\theta(\phi)$ integration.

Thus, for any given value of radial coordinate $r$ (and fixed $\theta$ and $\phi$ ), the Coulomb potential can be easily calculated just by evaluating the $9^{\text {th }}$ degree polynomial in $r$. It is found that this interpolation procedure is very accurate. The maximum percentage difference between the fitted and the exact Coulomb potentials is $0.4 \%$ for a highly deformed nucleus.

\section{VARIATION OF WIGNER-KIRKWOOD ENERGIES WITH DEFORMATION PARAMETERS}

A sample WK calculation is performed for a system of 126 neutrons and 82 protons. The variation of the ThomasFermi energy and of the different correction terms as a function of the quadrupole deformation parameter $\beta_{2}$ is presented in Figs. 2(a)-2(f). The other two deformation parameters, $\beta_{4}$ and $\gamma$, are set to zero in this test case. The partial contributions to the WK energy are plotted separately for protons and neutrons. It is found that all the correction terms vary smoothly as a function of deformation. As expected, the value of the contributions from the $\hbar^{2}$ and $\hbar^{4}$ terms to the averaged energy decreases rapidly. It is found that the proton and neutron Thomas-Fermi energies have opposite trends with respect to increasing $\beta_{2}$. If Coulomb potential is suppressed, it is found that the Thomas-Fermi energies for protons follow the same trend as those for the neutrons. Further, it is interesting to note that comparatively, the variation in the secondorder corrections with respect to deformation parameters is stronger than that in the Thomas-Fermi energies $(\sim 10 \%$ for second-order corrections and $\sim 3 \%$ for Thomas-Fermi energies).

Next, the variation of the Thomas-Fermi energy and of the correction terms as a function of the hexadecapole deformation parameter $\beta_{4}$ is plotted in Figs. 3(a)-3(f). Here, $\beta_{2}$ is taken to be 0.2 and $\gamma$ is set to zero. It is seen that, again, the different energies vary smoothly as a function of $\beta_{4}$. The Thomas-Fermi energy for protons is found to have very little variation with respect to the $\beta_{4}$ deformation parameter. In contrast, the corresponding energies for neutrons have a stronger dependence on $\beta_{4}$. The same behavior is also observed in the corresponding quantum mechanical energies. It is found that the proton and neutron Thomas-Fermi energies have a very similar behavior if the Coulomb potential is suppressed. Furthermore, to check if this conclusion depends on the value of $\beta_{2}$, the analysis is repeated for $\beta_{2}=0.4$, and the same conclusion is found to emerge.

The behavior of the Thomas-Fermi energies for protons in the above cases (Figs. 2 and 3) seems to be due to the Coulomb potential. In the case of variation with respect to $\beta_{2}$, qualitatively it can be expected that with increasing quadrupole deformation, protons are pulled apart and Coulomb repulsion decreases, thereby making the system more bound. The $\beta_{4}$ deformation also affects the proton distribution, but, as expected, the effect of hexadecapole deformation is less prominent in comparison with that of quadrupole deformation. Thus, the repulsion among protons does decrease with increasing $\beta_{4}$, but the decrease is not large enough to make the system more bound with larger $\beta_{4}$.

By keeping $\beta_{2}$ and $\beta_{4}$ fixed, if the parameter $\gamma$ is varied, then it is found that the resulting energies are independent of the sign of $\gamma$. Moreover, the $\gamma$ dependence of the WK energies is found to be rather weak. Therefore, here we do not present these results explicitly.

The fourth-order calculation for protons is very timeconsuming. Typically, it takes tens of minutes to do a complete WK calculation. Most of the runtime is consumed by particle number determination and the fourth-order calculations for protons. Thus, it is necessary to find an accurate approximation scheme for the fourth-order calculation for protons. Because in the nuclear interior, the Coulomb potential has approximately a quadratic nature (see Fig. 1), it is expected that the Coulomb potential will have small influence on the fourthorder calculations (note that one needs higher-order derivatives in the fourth-order energy calculations). One may therefore drop the Coulomb potential completely from the fourth-order corrections; we shall refer to this approximation as "quadratic approximation." This approximation has been checked explicitly by performing exact fourth-order calculations for protons. The maximum difference between the WK energies obtained by using exact calculation and the quadratic approximation is found to be of the order $100 \mathrm{keV}$ for 82 protons. It turns out that the difference between the quadratic approximation and exact calculation decreases with decreasing charge number. This approximation can be improved by keeping the Laplacian of the Coulomb potential in the fourth-order contribution [i.e., the terms of the form $\left(\nabla^{2} V\right)^{2}$ and $\nabla^{4} V$ in Eq. (17)]. This means that, for protons, only the term $\nabla^{2}(\nabla V)^{2}$ is dropped from Eq. (17). It is found that with this modification, the value of the fourth-order correction energy for the mean-field part for protons almost coincides with the value obtained by taking all of the derivatives of the Coulomb potential into account. This helps in reducing the total runtime further. Thus, effectively, with the interpolation for the Coulomb potential as discussed before (see Sec. IV), and the approximations introduced in the fourth-order correction terms for protons in the present section, the runtime reduces from tens of minutes to just about $2 \mathrm{~min}$, without affecting the desired accuracy of the calculations. 

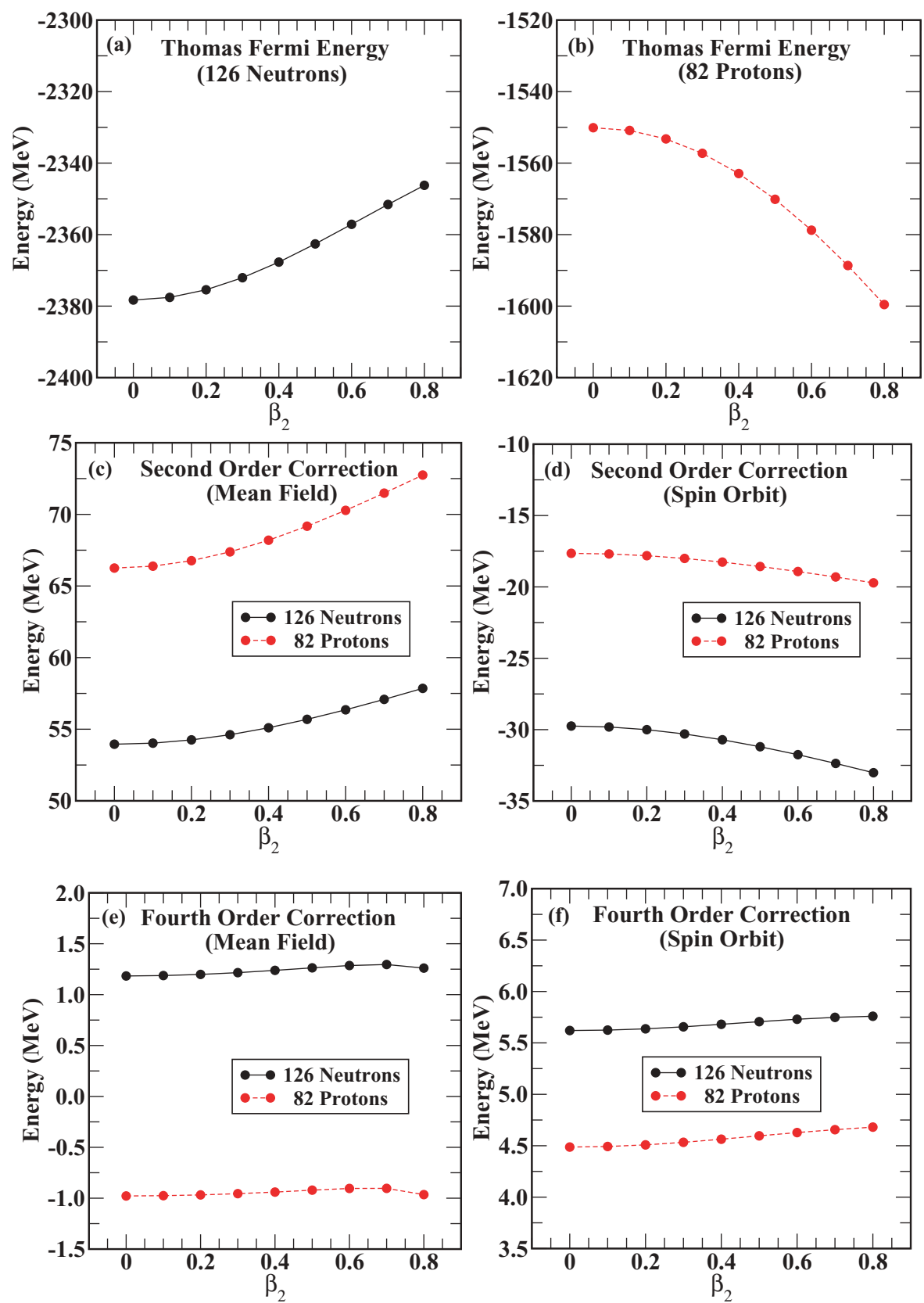

FIG. 2. (Color online) WignerKirkwood energies for 126 neutrons and 82 protons as a function of $\beta_{2}$. Here, $\beta_{4}=0$ and $\gamma=0$. The Thomas-Fermi energies, second-order corrections, and fourth-order corrections are shown in the top, middle, and bottom panels, respectively.

\section{WIGNER-KIRKWOOD SHELL CORRECTIONS AND COMPARISON WITH STRUTINSKY CALCULATIONS}

The equivalence between WK and the Strutinsky shell correction was actually summarized by Strutinsky himself in his earliest paper [16] and pointed out early in the 1970s by Tyapin [49] and Gross [50]. Numerically, it has been demonstrated that the WK and Strutinsky shell corrections are close to each other [20,51]. More recently, it has been shown [52] that the Strutinsky level density is an approximation to the semiclassical WK level density. For illustration, we present and discuss the WK and the corresponding Strutinsky shell corrections for the chains of $\mathrm{Pb}, \mathrm{Gd}$, and $\mathrm{Dy}$ isotopes. For the sake of completeness, we first present and discuss the essential features of the Strutinsky smoothing scheme.
According to the Strutinsky smoothing scheme, the smooth level density for a one-body Hamiltonian is given [53]:

$$
g_{s t}(\epsilon)=\frac{1}{\gamma \sqrt{\pi}} \sum_{i=1}^{\infty} e^{-\left(\epsilon-\epsilon_{i}\right)^{2} / \gamma^{2}} \sum_{j=1}^{N_{s}} S_{j} H_{j}\left(\frac{\epsilon-\epsilon_{i}}{\gamma}\right),
$$

where $\epsilon_{i}$ are the single-particle energies calculated by diagonalizing the Hamiltonian matrix. The smoothing constant $\gamma$ is taken to be of the order of $\hbar \omega_{0}\left(\hbar \omega_{0}=1.2 \times 41 A^{-1 / 3}\right) . N_{s}$ is the smoothing order, and is assumed to be equal to 6 in the present work; $H_{j}$ are the Hermite polynomials; and $S_{j}$ is a constant, defined as [53]

$$
\begin{array}{rlrl}
S_{j} & =\frac{(-1)^{j / 2}}{2^{j}(j / 2) !}, & & \text { for } \quad j \text { even, } \\
& =0, & \text { for } j \text { odd }
\end{array}
$$



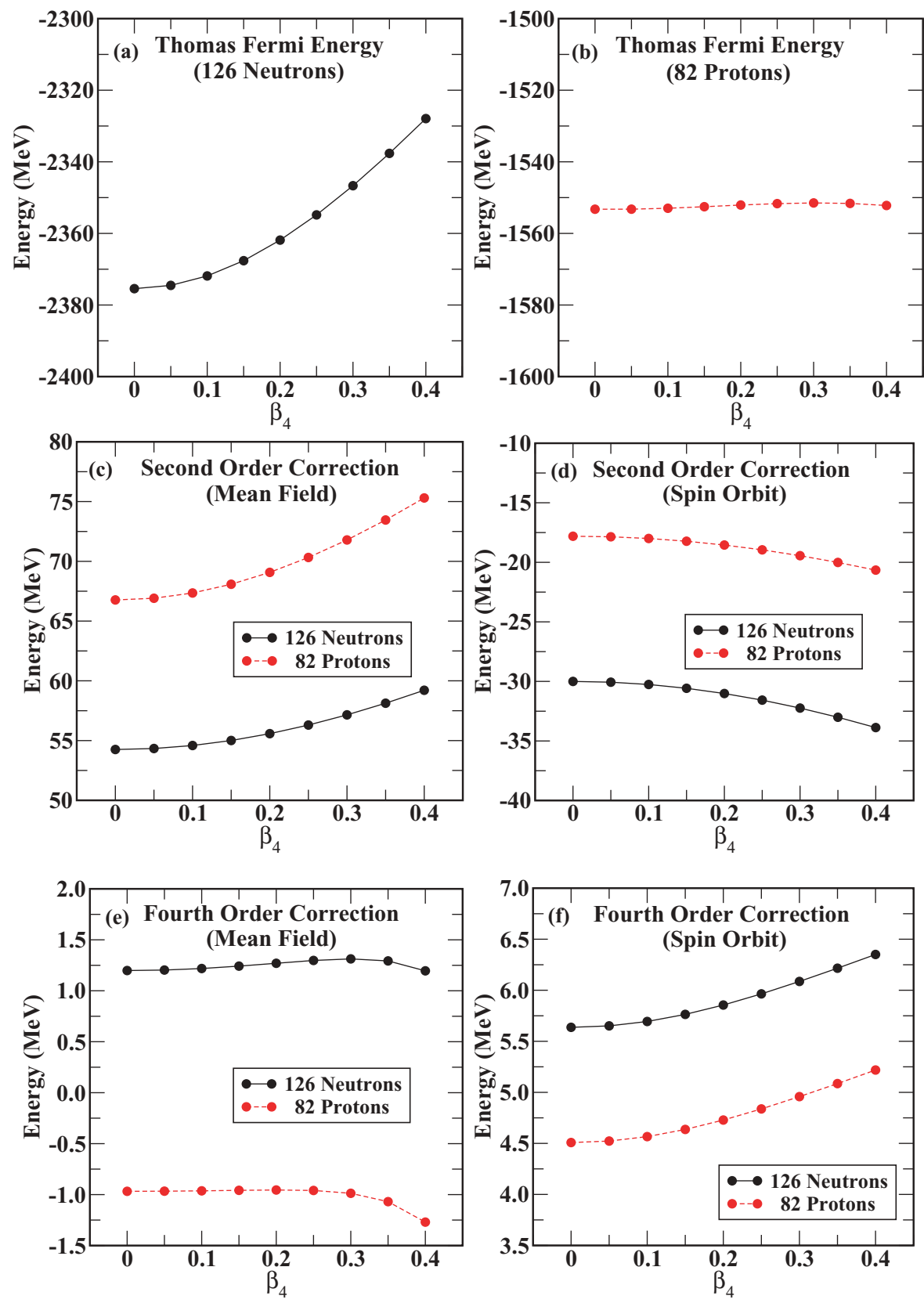

FIG. 3. (Color online) WignerKirkwood energies for 126 neutrons and 82 protons as a function of $\beta_{4}$. Here, $\beta_{2}=0.2$ and $\gamma=0$. The Thomas-Fermi energies, second-order corrections, and fourth-order corrections are shown in the top, middle, and bottom panels, respectively.
The Strutinsky shell correction is given:

$$
E_{\mathrm{St}}=\sum_{i=1}^{N_{n}} \epsilon_{i}-\int_{-\infty}^{\bar{\lambda}} \epsilon g_{s t}(\epsilon) d \epsilon
$$

where $N_{n}$ is the number of nucleons. This, upon substituting the expression for $g_{s t}$, yields [53],

$$
\begin{aligned}
E_{s t}= & \sum_{i=1}^{N_{n}} \epsilon_{i}-\sum_{j=0}^{\infty}\left\{\frac{\epsilon_{j}}{2}\left[1+\operatorname{erf}\left(\bar{u}_{j}\right)\right]-\frac{\gamma e^{-\bar{u}_{j}^{2}}}{2 \sqrt{\pi}}-\frac{e^{-\bar{u}_{j}^{2}}}{\sqrt{\pi}}\right. \\
& \left.\times \sum_{k=1}^{N_{s}} S_{k}\left[\frac{\gamma}{2} H_{k}\left(\bar{u}_{j}\right)+\epsilon_{j} H_{k-1}\left(\bar{u}_{j}\right)+k \gamma H_{k-2}\left(\bar{u}_{j}\right)\right]\right\},
\end{aligned}
$$

where

$$
\bar{u}_{j}=\frac{\bar{\lambda}-\epsilon_{j}}{\gamma} .
$$

Here, $\bar{\lambda}$ is the chemical potential, calculated iteratively from the particle number condition. The error integral erf $(x)$ is defined as

$$
\operatorname{erf}(x)=\frac{2}{\sqrt{\pi}} \int_{0}^{x} e^{-z^{2}} d z .
$$

It should be noted that the Strutinsky procedure described here uses positive energy states generated by diagonalizing the Hamiltonian matrix, and not by taking resonances into account and smoothing them. Furthermore, in practice, the summations defined above do not extend up to infinity, but are cut off at a suitable upper limit. The limit is chosen in such a way that 

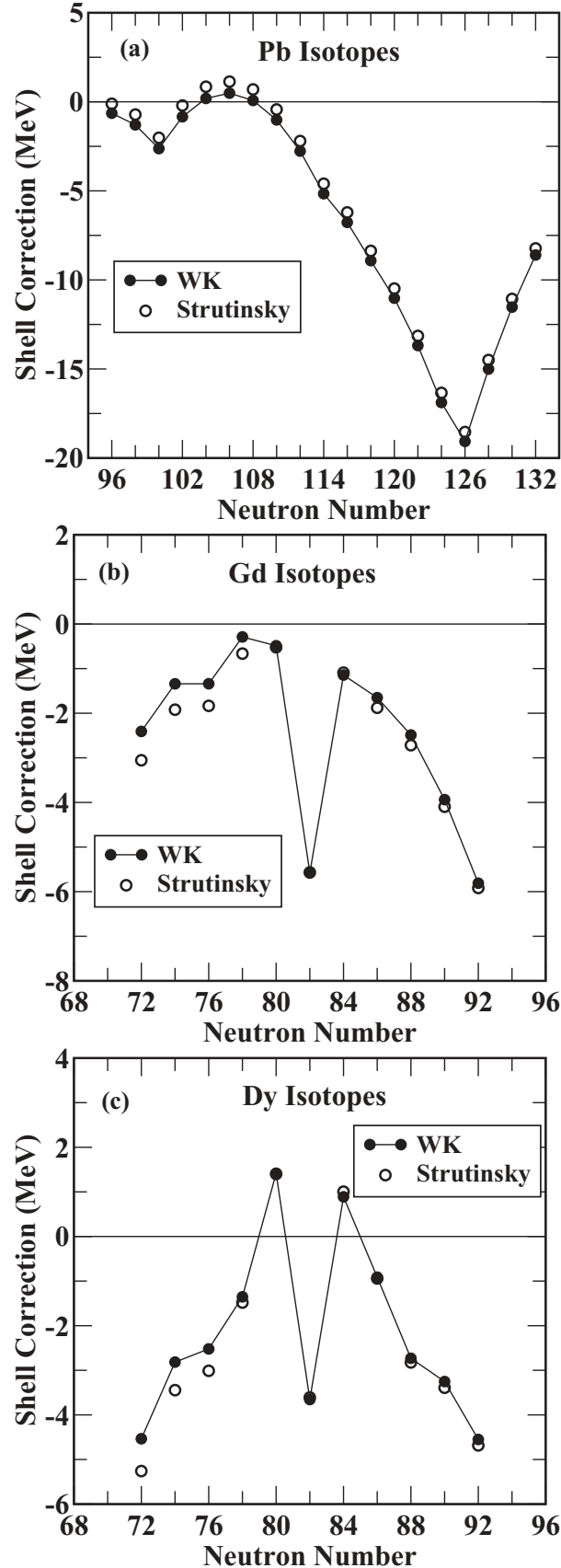

FIG. 4. WK and the corresponding Strutinsky shell corrections for $\mathrm{Pb}$ (top), Gd (middle), and Dy (bottom) isotopes.

all the states up to $\sim 4 \hbar \omega_{0}$ are included in the sum. It has been shown that the uncertainty in the Strutinsky shell corrections obtained this way is typically of the order of $0.5 \mathrm{MeV}$ [53]. For lighter nuclei, however, it has been concluded [53] that this uncertainty is larger.

The total WK shell correction for the chain of even-even Lead isotopes $\left({ }^{178-214} \mathrm{~Pb}\right)$ is plotted in Figs. 4(a)-4(c), along with the corresponding values obtained by using the Strutinsky smoothing method. It is found that both the WK and Strutinsky results exhibit very similar trends. As expected, there is a prominent minimum observed for ${ }^{208} \mathrm{~Pb}$, indicating the occurrence of shell closure. The WK and Strutinsky shell corrections slightly differ from each other. The difference is not a constant, and is found to be increasing slowly toward the more neutron-deficient Lead isotopes.

Next we plot the calculated (WK) and the corresponding Strutinsky shell corrections for the chains of even-even Gd and Dy isotopes, with neutron numbers ranging from 72 to 92 . Apart from ${ }^{144,146,148} \mathrm{Gd}$ and ${ }^{146,148,150} \mathrm{Dy}$, the rest of the nuclei considered here are known to be deformed [12]. For this test run, we adopt the deformation parameters from the Möller-Nix compilation [12]. It is seen that the $\mathrm{WK}$ and the corresponding Strutinsky shell corrections agree with each other, within a few hundred keVs. The prominent minimum at shell closure at neutron number 82 is clearly seen. In these cases as well, the difference between the two calculations is not a constant. It is larger in the neutron-deficient region, and becomes smaller as the neutron number increases.

\section{LIPKIN-NOGAMI PAIRING MODEL}

The pairing correlations, important for the open shell nuclei, are often taken into account within the framework of the BCS model. The BCS model, however, has two serious shortcomings: 1) particle number fluctuation (the BCS wave functions are not particle number eigenstates), and 2) there may exist critical values of the pairing strength, below which the BCS equations may not have any nontrivial solutions. To overcome these difficulties, Lipkin, Nogami, and co-workers proposed to minimize the expectation value of the model Hamiltonian [29-31],

$$
\hat{\mathcal{H}}=\hat{H}-\lambda_{1} \hat{N}-\lambda_{2} \hat{N}^{2},
$$

by determining $\lambda_{1}$ and $\lambda_{2}$ using certain conditions. Here, $\hat{H}$ is the pairing Hamiltonian and $\hat{N}$ is the particle number operator. Minimization of the expectation value of $\hat{H}-\lambda_{1} \hat{N}$ leads to the usual BCS model, with $\lambda_{1}$ determined from the particle number condition. Thus, in Eq. (50) above, the quantity $\lambda_{1}$ is a Lagrange multiplier, but the particle number fluctuation constant $\lambda_{2}$ is not.

In practice, the LN calculation is carried out by assuming a constant pairing matrix element, $G$. For a given nucleus (assumed to be even-even for simplicity), one considers $N_{h}$ doubly degenerate states below, and $N_{p}$ doubly degenerate states above the Fermi level. These states contain $\mathcal{N}$ nucleons. In practice, one takes $N_{h}=N_{p}=N / 2$ or $Z / 2$, depending on whether it is being applied to neutrons or protons. The occupation probabilities $v_{k}^{2}$, the pairing gap $\Delta$, the chemical potential $\lambda\left(=\lambda_{1}+2 \lambda_{2}(\mathcal{N}+1)\right.$; see Ref. [31]), and the constant $\lambda_{2}$ are determined iteratively using the conditions $[13,31]$ :

$$
\begin{aligned}
\mathcal{N} & =2 \sum_{k} v_{k}^{2} \\
\Delta & =G \sum_{k} u_{k} v_{k},
\end{aligned}
$$

such that

$$
v_{k}^{2}=\frac{1}{2}\left\{1-\frac{\varepsilon_{k}-\lambda}{\left[\left(\varepsilon_{k}-\lambda\right)^{2}-\Delta^{2}\right]^{1 / 2}}\right\},
$$


and

$$
\varepsilon_{k}=E_{k}+\left(4 \lambda_{2}-G\right) v_{k}^{2},
$$

where $E_{k}$ are the single-particle energies and $u_{k}^{2}=1-v_{k}^{2}$. The particle number fluctuation constant $\lambda_{2}$ is given:

$$
\lambda_{2}=\frac{G}{4}\left[\frac{\left(\sum_{k} u_{k}^{3} v_{k}\right)\left(\sum_{k} u_{k} v_{k}^{3}\right)-\sum_{k} u_{k}^{4} v_{k}^{4}}{\left(\sum_{k} u_{k}^{2} v_{k}^{2}\right)^{2}-\sum_{k} u_{k}^{4} v_{k}^{4}}\right] .
$$

The pairing matrix element $G$ is calculated by the Möller-Nix prescription [13]:

$$
\frac{2}{G}=\bar{\rho}_{L} \ln \left\{\sqrt{a_{2}^{2}+\bar{\Delta}^{2}}+a_{2}\right\}-\bar{\rho}_{L} \ln \left\{\sqrt{a_{1}^{2}+\bar{\Delta}^{2}}+a_{1}\right\} .
$$

Here, $\bar{\rho}_{L}=g_{\mathrm{WK}} / 2$ is the Wigner-Kirkwood averaged level density [see Eq. (15)]. A factor of 2 appears because each quantal level here has a degeneracy of 2 . The level density is evaluated at Fermi energy; $a_{2}=\mathcal{N} / 2 \bar{\rho}_{L}, a_{1}=-\mathcal{N} / 2 \bar{\rho}_{L}$, and $\bar{\Delta}$ is the average pairing gap, taken to be $3.3 / \mathcal{N}^{1 / 2}$ [13].

The ground-state energy within the LN model is given:

$$
E_{g}=2 \sum_{k} v_{k}^{2} E_{k}-\frac{\Delta^{2}}{G}-G \sum_{k} v_{k}^{4}-4 \lambda_{2} \sum_{k} u_{k}^{2} v_{k}^{2} .
$$

The pairing correlation energy, $E_{\text {pair }}$ is obtained by subtracting the ground-state energy in the absence of pairing from Eq. (57):

$$
E_{\text {pair }}=E_{g}-2 \sum_{k} E_{k}-G \mathcal{N} / 2
$$

\section{CALCULATION OF BINDING ENERGIES}

As an illustrative example, we now present and discuss the calculated binding energies (in this paper, we take binding energies as negative quantities) for 367 even-even, even-odd, odd-even, and odd-odd spherical nuclei. These nuclei are predicted to be spherical or nearly spherical $\left(\beta_{2}<0.05\right)$ in the Möller-Nix calculations [12] and include ${ }^{38-52} \mathrm{Ca},{ }^{42-54} \mathrm{Ti}$, ${ }^{100-134} \mathrm{Sn}$, and ${ }^{178-214} \mathrm{~Pb}$. The detailed list of nuclei considered in the present fit can be found in Ref. [54]. Of course, it is known that the prediction of sphericity does depend to some extent on the details of the density functional employed [55]. Therefore, it may so happen that some of the nuclei assumed to be spherical here may actually turn out to be slightly deformed when energy minimization is carried out on the grid of deformation parameters.

Our calculation proceeds in the following steps. For each nucleus, the quantum mechanical and WK energies are calculated as described in Appendix A by using 15 deformed harmonic oscillator shells. As it is shown in this appendix, the correction due to the truncation of the basis is actually small and its effect can be absorbed by the liquid-drop model (LDM) parameters (see the following). This then yields values of the shell corrections $(\delta E)$ for these nuclei. The pairing energies $\left(E_{\text {pair }}\right)$ are then calculated using the Lipkin-Nogami scheme [29-31] described previously in the same potential well where the shell correction is computed. These two pieces constitute the microscopic part of the binding energy. The macroscopic part of the binding energy $\left(E_{\mathrm{LDM}}\right)$ is obtained
TABLE I. Values of the liquid drop parameters obtained through the $\chi^{2}$ minimization.

\begin{tabular}{lccc}
\hline \hline Quantity & Value & Quantity & Value \\
\hline$a_{v}$ & $-15.841(\mathrm{MeV})$ & $a_{s}$ & $19.173(\mathrm{MeV})$ \\
$k_{v}$ & -1.951 & $k_{S}$ & -2.577 \\
$r_{0}$ & $1.187(\mathrm{fm})$ & $C_{4}$ & $1.247(\mathrm{MeV})$ \\
\hline \hline
\end{tabular}

from the liquid drop formula. Thus, for a given nucleus with $Z$ protons and $N$ neutrons (mass number $A=N+Z$ ), the binding energy in the Mic-Mac picture is given:

$$
E(N, Z)=E_{\mathrm{LDM}}+\delta E+E_{\text {pair }} .
$$

The liquid drop part of binding energy is chosen:

$$
\begin{aligned}
E_{\mathrm{LDM}}= & a_{v}\left[1+\frac{4 k_{v}}{A^{2}} T_{z}\left(T_{z}+1\right)\right] A \\
& +a_{s}\left[1+\frac{4 k_{s}}{A^{2}} T_{z}\left(T_{z}+1\right)\right] A^{2 / 3} \\
& +\frac{3 Z^{2} e^{2}}{5 r_{0} A^{1 / 3}}+\frac{C_{4} Z^{2}}{A},
\end{aligned}
$$

where the terms, respectively, represent volume energy, surface energy, Coulomb energy, and correction to Coulomb energy due to surface diffuseness of charge distribution. The coefficients $a_{v}, a_{s}, k_{v}, k_{s}, r_{0}$, and $C_{4}$ are free parameters; $T_{z}$ is the third component of isospin, and $e$ is the electronic charge. The free parameters are determined by minimizing the $\chi^{2}$ value in comparison with the experimental energies:

$$
\chi^{2}=\frac{1}{n} \sum_{j=0}^{n}\left[\frac{E\left(N_{j}, Z_{j}\right)-E_{\mathrm{expt}}^{(j)}}{\Delta E_{\mathrm{expt}}^{(j)}}\right]^{2},
$$

where $E\left(N_{j}, Z_{j}\right)$ is the calculated total binding energy for the given nucleus, $E_{\text {expt }}^{(j)}$ is the corresponding experimental value [56], and $\Delta E_{\text {expt }}^{(j)}$ is the uncertainty in $E_{\text {expt }}^{(j)}$. In the present fit, for simplicity, $\Delta E_{\text {expt }}^{(j)}$ is set to $1 \mathrm{MeV}$. The

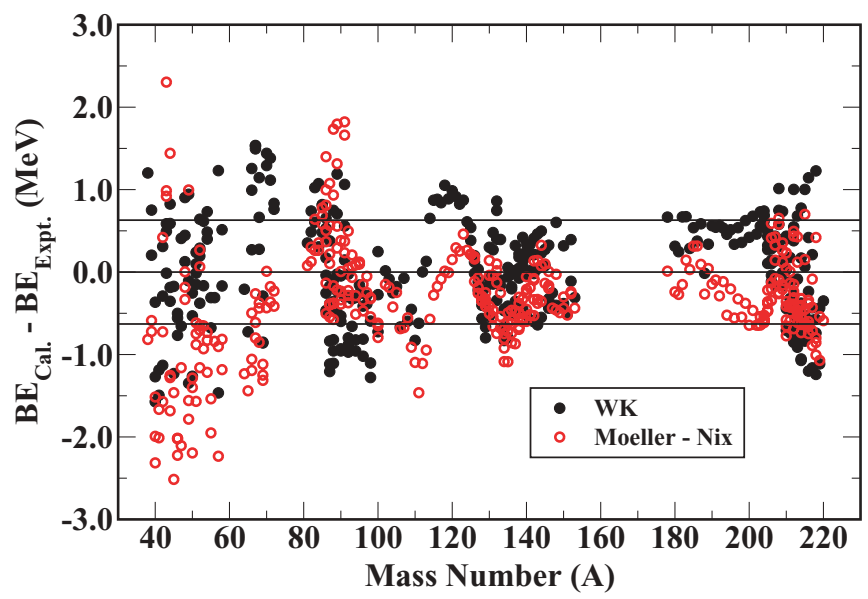

FIG. 5. (Color online) Difference between the calculated and the experimental [56] binding energies. The corresponding differences obtained for the Möller-Nix values of binding energies [13] are also presented. 

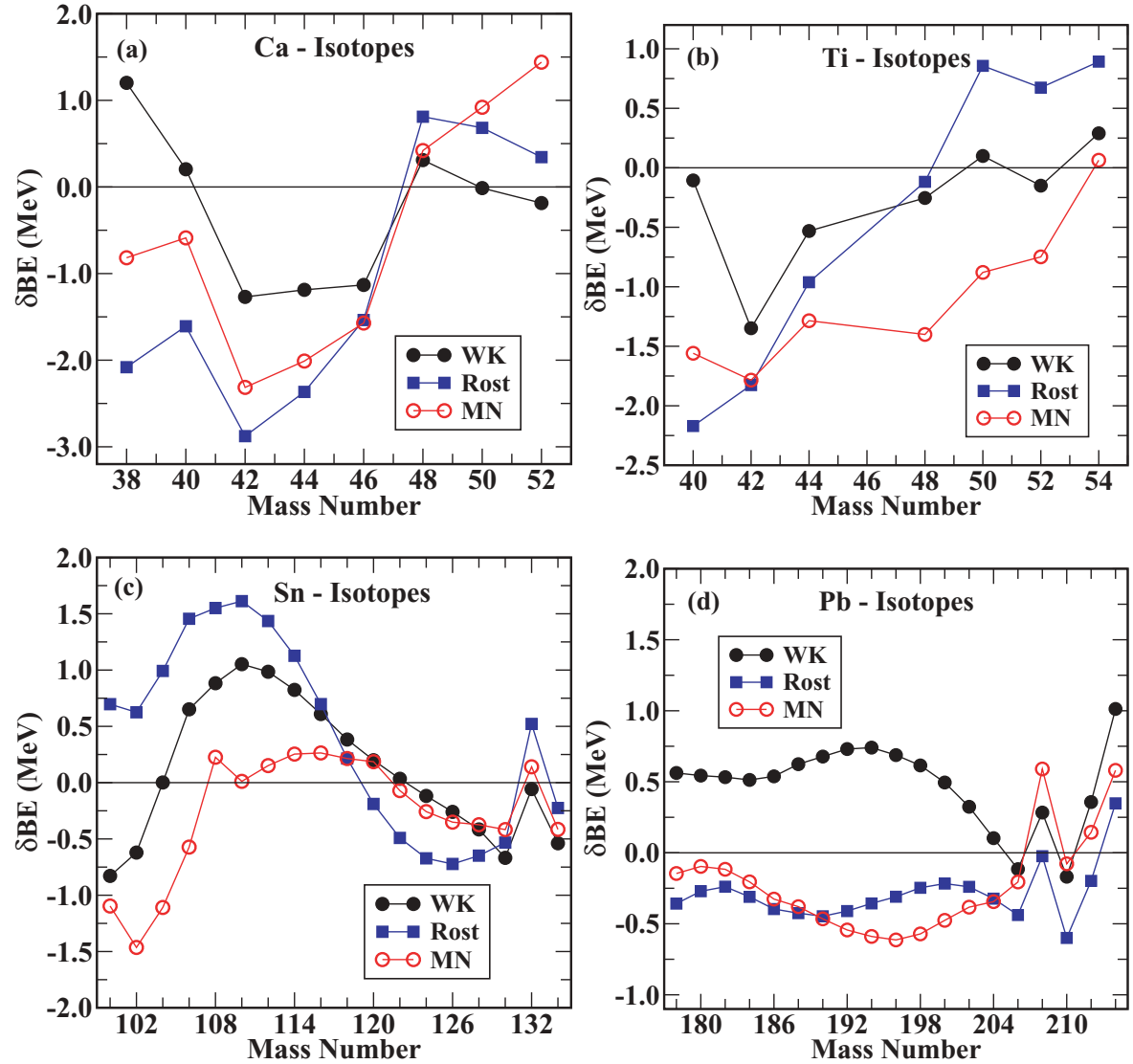

FIG. 6. (Color online) Difference between calculated binding energies and experiment [56]. Results are shown for the present calculation (WK), for the Möller-Nix values (MN), and using the Rost parameters in the Woods-Saxon form factors as described in the text. minimization is achieved using the well-known LevenbergMarquardt algorithm [57,58].

For the set of nuclei considered here, the rms deviation in binding energies turns out to be $630 \mathrm{keV}$, which, indeed, is gratifying. The rms deviation obtained for the same nuclei with the Möller-Nix mass formula turns out to be $741 \mathrm{keV}$. The liquid drop parameters are presented in Table I. Clearly, the obtained values of the parameters are reasonable. The detailed table containing the nuclei considered in the present fit, and the corresponding calculated and experimental [56] binding energies may be found in Ref. [54]. We point out here that if the effect of truncation of the harmonic oscillator basis is taken into account, the parameters reported in Table I change only very little and the rms corresponding to the 367 nuclei considered in our analysis is practically unaffected.

To examine the quality of the fit further, first we plot the difference between the fitted and the corresponding experimental [56] binding energies for the 367 nuclei as a function of the mass number $A$ in Fig. 5. The corresponding differences obtained for the Möller-Nix [13] values of binding energies are also plotted in the same figure for comparison. It is amply clear from the figure that the fitted binding energies are close to the experiment (within $1 \mathrm{MeV}$ ). Overall, the quality of the present fit is slightly better than that of the Möller-Nix fit (the rms deviations, respectively, are 630 and $741 \mathrm{keV}$ ). Particularly for the lighter nuclei, the present calculations are comparatively closer to the experiment.
Next, the difference between the calculated and the corresponding experimental [56] binding energies (denoted by $\mathrm{WK}$ ) for the $\mathrm{Ca}, \mathrm{Ti}, \mathrm{Sn}$, and $\mathrm{Pb}$ isotopes considered in this fit are presented in Figs. 6(a)-6(d). The differences obtained by using the Möller-Nix [13] values of binding energies (denoted by $\mathrm{MN}$ ) are also shown there for comparison. It can be seen that the present calculations agree well with the experiment. It is found that the differences vary smoothly as a function of mass number: the exceptions being the doubly closed shell nuclei ${ }^{48} \mathrm{Ca},{ }^{132} \mathrm{Sn}$, and ${ }^{208} \mathrm{~Pb}$, where a kink is observed. The overall behavior of the differences is somewhat smoother than that obtained by using the values of Möller and Nix.

To investigate the effect of the parameters of the singleparticle potential, we make a refit of the liquid drop parameters by using the Rost parameters [59] in the microscopic part of the binding energy computed in the WK approximation. That is, we calculate the shell corrections and pairing energies employing the Rost parameters for the Woods-Saxon form factors, and then fit the liquid drop parameters again for the same set of nuclei. The rms deviation obtained in this case (1.14 MeV for even-even nuclei) is much worse that the one obtained for the parameters mentioned in Sec. III (the rms deviation obtained for this potential is around $0.680 \mathrm{MeV}$ ), which is amply clear from the figure. It is well known that the Rost parameters have very large half-density radii. As a consequence, the values of the moment of inertia and rms radii obtained by using these parameters deviate strongly from the corresponding experimental values. On the contrary, the parametrization used in the present analysis (see Sec. III), 

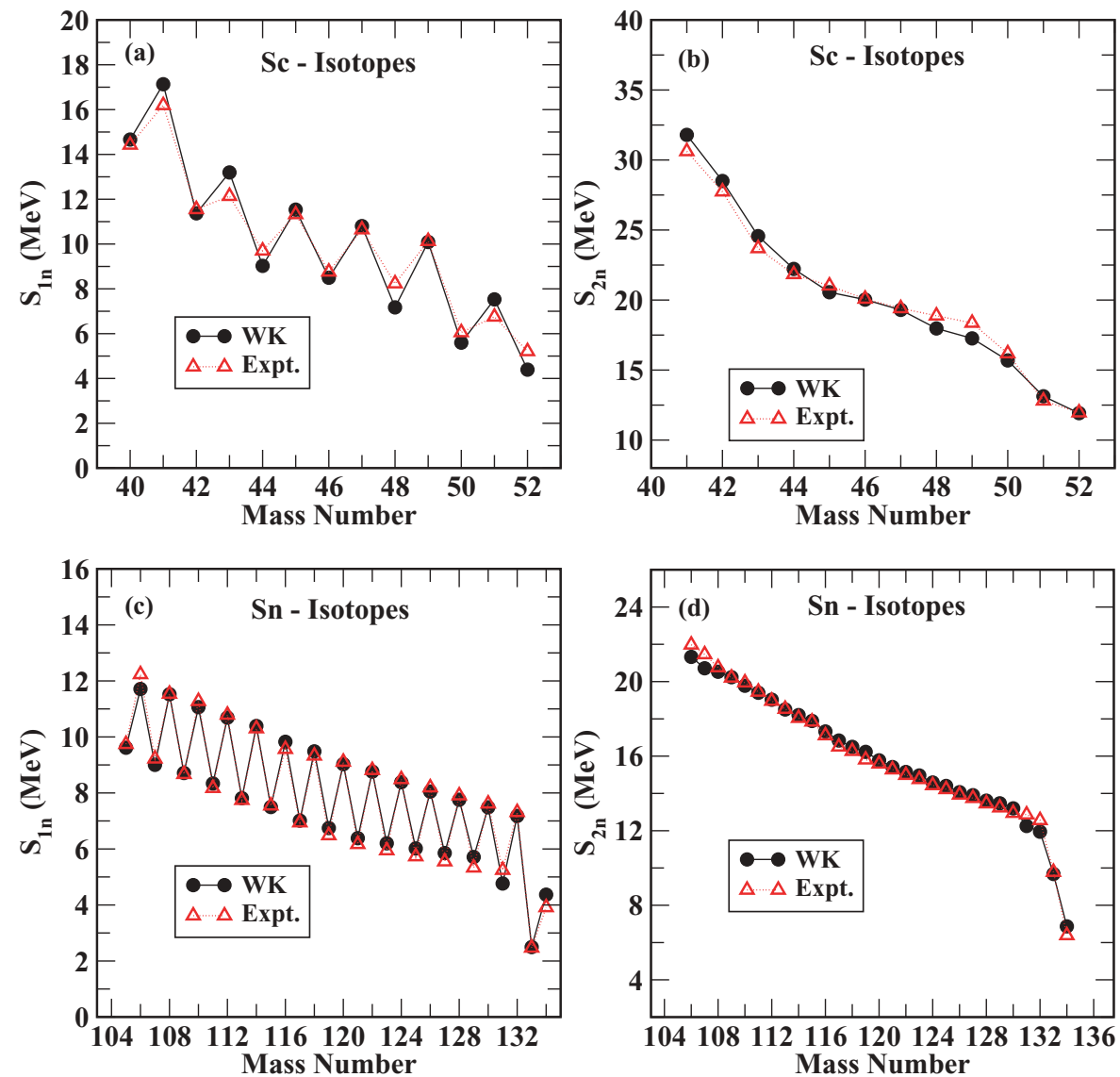

FIG. 7. (Color online) The calculated and the corresponding experimental [56] one- and two-neutron separation energies for $\mathrm{Sc}, \mathrm{Sn}$, and $\mathrm{Pb}$ isotopes.
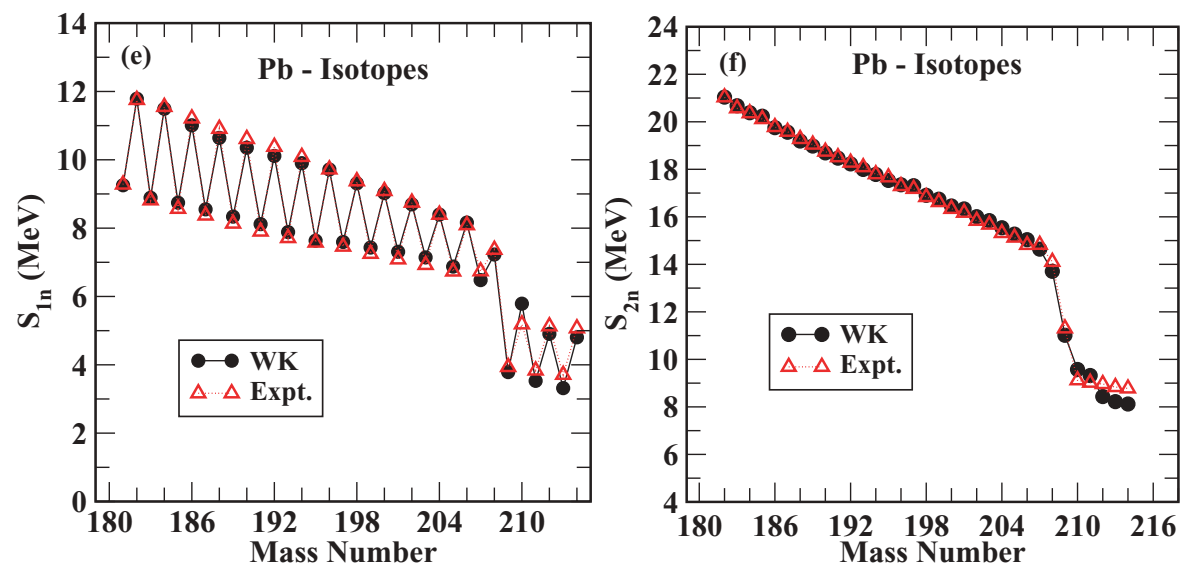

yields reasonable values of moments of inertia and radii. Thus, overall, this potential is more realistic than the Rost potential. This is reflected in the calculated binding energies as well, showing clearly that the choice of the single-particle potential (or, in other words, the parameters) is indeed important for reliable predictions of binding energies (and hence the masses).

Single- and two-neutron separation energies $\left(S_{1 n}\right.$ and $\left.S_{2 n}\right)$ are crucial observables. They are obtained by calculating binding energy differences between pairs of isotopes differing by one and two neutron numbers, respectively. The single neutron separation energies govern asymptotic behavior of the neutron density distributions [60]. They exhibit odd-even staggering along an isotopic chain, indicating that the isotopes with an even number of neutrons are more bound than the neighboring isotopes with an odd number of neutrons. The systematics of $S_{2 n}$ primarily reveals the shell structure in an isotopic chain. The correct prediction of these separation energies is crucial for determination of the neutron drip lines. The calculated $S_{1 n}$ and $S_{2 n}$ values for $\mathrm{Sc}, \mathrm{Sn}$, and $\mathrm{Pb}$ isotopes are displayed in Figs. 7(a)-7(f). The corresponding experimental values of $S_{1 n}$ and $S_{2 n}$ [56] are also plotted for comparison. The agreement between calculations and experiment is found to be excellent. The odd-even staggering is nicely reproduced. The shell closures at ${ }^{132} \mathrm{Sn}$ and ${ }^{208} \mathrm{~Pb}$ are clearly visible both in single- and two-neutron separation energies. At a finer level, however, a marginal underestimation of the shell gap at the neutron number 82 (126) is observed in ${ }^{132} \mathrm{Sn}\left({ }^{208} \mathrm{~Pb}\right)$. Finally, we remark that the calculated 
single- and two-proton separation energies are also found to be in close agreement with the experiment.

The results presented in this section indicate that the present calculations of binding energies, indeed, are reliable.

\section{SUMMARY AND FUTURE OUTLOOK}

In the present work, we intend to carry out reliable mass calculations for the nuclei spanning the entire periodic table. For this purpose, we employ the "microscopic-macroscopic" framework. The microscopic component has two ingredients: the shell correction energy and the pairing energy. The pairing energy is calculated by using the well-known Lipkin-Nogami scheme. To average out the given one-body Hamiltonian (and hence find the shell corrections, given the total quantum mechanical energy of the system), we use the semiclassical Wigner-Kirkwood expansion technique. This method does not use the detailed single-particle structure, as in the case of the conventional Strutinsky smoothing method. In addition to the bound states, the Strutinsky scheme requires the contributions coming in from the continuum as well. Treating the continuum is often tricky, and in most of the practical calculations, the continuum is taken into account rather artificially, by generating positive energy states by means of diagonalization of the Hamiltonian matrix. For neutronrich and neutron-deficient nuclei, the contribution from the continuum becomes more and more important as the Fermi energy becomes smaller (less negative). Uncertainty in the conventional Strutinsky scheme thus increases as one goes away from the line of stability. It is therefore expected that the Wigner-Kirkwood method will be a valuable and suitable option especially for nuclei lying far away from the line of stability.

We now summarize our observations and future perspectives:

(i) Semiclassical averaging of a realistic one-body Hamiltonian using the Wigner-Kirkwood expansion of the partition function correct up to the fourth order in $\hbar$ is carried out for the deformed systems, both for protons and neutrons. The spin orbit as well as Coulomb potentials are explicitly taken into account.

(ii) The smooth energies thus obtained are investigated in detail as a function of three deformation parameters: $\beta_{2}, \beta_{4}$, and $\gamma$. As expected, the energies corresponding to the leading-order term in the expansion as well as the correction terms vary smoothly as a function of deformation parameters.

(iii) Differences between the quantum mechanical and the corresponding averaged energies yield the shell corrections. These, along with the pairing energies obtained by using the Lipkin-Nogami scheme, constitute the "microscopic" part of the nuclear binding energy in the "Mic-Mac" picture. Using a simple liquid drop ansatz with six adjustable parameters, it is demonstrated that the present approach, indeed, is feasible and very promising. For the test case presented here, comprising 367 spherical nuclei, the rms deviation of the predicted binding energies from the experimental values turns out to be $630 \mathrm{keV}$. We have also checked that the truncation error of the harmonic oscillator basis is negligible in light nuclei but it is more significant in heavy nuclei. However, its impact on the LDM parameters of the macroscopic part of the model and on the variation of the rms deviation is practically negligible.

(iv) The importance of the one-body potential in reliable estimations of nuclear binding energies is explicitly demonstrated. It should be noted that the Woods-Saxon parameters used in this work have been fitted for the Coulomb potential calculated by using the uniform density (sharp surface) approximation. The Coulomb potential we use is obtained from folding Woods-Saxon density profile with the Coulomb interaction. Therefore, before performing the large-scale calculations, we intend to make a refit to the Woods-Saxon potential, with the Coulomb potential obtained from folding.

(v) Having established the feasibility of the present approach, we now intend to extend our binding energy calculations to deformed nuclei. For this purpose, we plan to minimize the binding energy on a mesh of deformation parameters to find the absolute minimum in the deformation space. Work along these lines is in progress.

\section{ACKNOWLEDGMENTS}

A.B. is thankful to KTH Stockholm and IPN Orsay for financial support. M.C. and X.V. were partially supported by the Consolider Ingenio 2010 Programme CPAN CSD200700042, Grant No. FIS2008-01661 from MEC and FEDER, and Grant No. 2009SGR-1289 from Generalitat de Catalunya.

\section{APPENDIX A: DETAILS OF QUANTUM MECHANICAL CALCULATIONS}

The one-body Hamiltonian defined in Eq. (2) is diagonalized in Harmonic oscillator basis to obtain the energy eigenfunctions and eigenvalues. The sum of eigenvalues up to the Fermi state gives total quantum mechanical energy of the system. In this work, we employ axially symmetric deformed harmonic oscillator basis. In the present work, we restrict the basis to 15 oscillator shells. The nature of deformation of the basis depends on the value of the deformation parameter $\gamma$. In particular:

(i) For $\gamma$ in the range from $-120^{\circ}$ to $-90^{\circ}$, the basis corresponds to $\gamma=-120^{\circ}$ (prolate shape).

(ii) For $\gamma$ in the range from $-90^{\circ}$ to $-30^{\circ}$, the basis corresponds to $\gamma=-60^{\circ}$ (oblate shape).

(iii) For $\gamma$ in the range from $-30^{\circ}$ to $+30^{\circ}$, the basis corresponds to $\gamma=0^{\circ}$ (oblate shape).

(iv) For $\gamma$ in the range from $+30^{\circ}$ to $+60^{\circ}$, the basis corresponds to $\gamma=60^{\circ}$ (oblate shape).

The oscillator frequencies required (one in $z$ direction, $\omega_{z}$, and the other for $X Y$ plane, $\omega_{\perp}$ ) are chosen employing the following two conditions $[43,61]$ : 
(i) The volume conservation condition,

$$
\omega_{\perp}^{2} \omega_{z}=\omega_{o}^{3}
$$

where $\omega_{o}$ is chosen such that

$$
\hbar \omega_{o}=f_{o} \frac{41}{A^{1 / 3}}, \quad \text { in } \mathrm{MeV},
$$

where $f_{o}$ is a factor chosen so as to ensure maximum stability of the solutions over a large range of deformation parameters [43]. In the present work, $f_{o}$ is taken to be equal to 1.2 .

(ii) The oscillator frequencies are also required to satisfy the following additional condition (sometimes referred to as "stretching"):

$$
\frac{\omega_{\perp}}{\omega_{z}}=\sqrt{\frac{2\left\langle z^{2}\right\rangle}{\left\langle\rho^{2}\right\rangle}},
$$

where $\rho^{2}=x^{2}+y^{2}$ and the average is calculated over the nuclear volume, defined by $r=r_{s}$ [see Eq. (21)].

As mentioned above, here we use the basis made of 15 oscillator shells. In principle, the basis set should be complete. The effect of truncation of basis is expected to be very small in the case of shell corrections obtained using the Strutinsky smoothing scheme. However, the truncation may have a non-negligible effect on the shell corrections obtained using Wigner-Kirkwood smoothed energies. We now explicitly demonstrate that even though this effect is indeed significant, particularly for heavier nuclei, its effect on the quality of the predicted binding energies is very small.

To begin with, we select a set of 132 nuclei, expected to be spherical, ranging from $\mathrm{Ca}$ isotopes to $\mathrm{Ra}$ isotopes. The quantum mechanical energy for these nuclei is obtained by using the standard basis expansion technique. The calculations are carried out for oscillator shells ranging from 10 to 19 . The ground-state energies per particle are then investigated as a function of the number of major shells used in the expansion. The results for ${ }^{208} \mathrm{~Pb},{ }^{132} \mathrm{Sn}$, and ${ }^{40} \mathrm{Ca}$ are presented in Fig. 8. Note that here we have plotted the absolute values of the ground-state energies per particle. The solid black curve represents the exact values. It is quite clear from the figure that the behavior of the ground-state energies per particle approximately follows:

$$
\varepsilon(N)=a_{0}\left\{1-\frac{a_{1}}{\cosh \left[a_{2}\left(N-a_{3}\right)\right]}\right\},
$$

where $\varepsilon(N)$ represents (negative of) ground-state energy per particle, $N$ is the number of shells, and $a_{j}$ 's are free parameters.

Indeed, it is found that this simple equation fits the calculated behavior of the ground- state energies per particle extremely well, the $\chi^{2}$ being of the order of $10^{-6}$ or even better. The desired value of ground-state energy for $N \rightarrow \infty$ is simply given by the parameter $a_{0}$.

Next, we carry out $\chi^{2}$ fit for all the 132 nuclei considered and deduce the parameter $a_{0}$ in each case. In the present work, we have used 15 shells for the quantum mechanical calculations. Therefore, it is desirable to deduce corrections for the total ground-state energy obtained in those cases. We

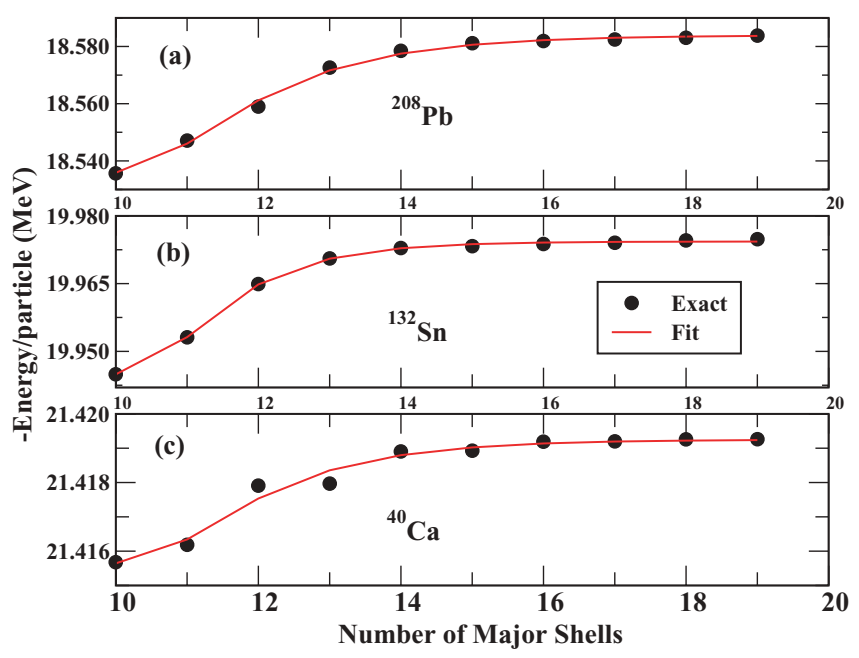

FIG. 8. (Color online) Exact and fitted ground-state energies for ${ }^{208} \mathrm{~Pb},{ }^{132} \mathrm{Sn}$, and ${ }^{40} \mathrm{Ca}$. Note that here the negative of ground-state energies is considered.

plot these corrections as a function of mass number in Fig. 9. The behavior of this energy as a function of mass number is found to be quite smooth. By inspection, we use the following ansatz for fitting $a_{0}$ as a function of mass number:

$$
a_{0}=c_{0} \sinh \left(c_{1} A\right) .
$$

It is found that, indeed, this ansatz fits the calculated $a_{0}$ very well (with standard error of the order 0.04; see the solid curve in Fig. 9). The parameters $c_{0}$ and $c_{1}$ turn out to be 0.01469 and 0.02087 , respectively.

Due to the functional form of $a_{0}$ selected here, the correction from the infinite basis for small mass numbers automatically becomes very small. Secondly, to check if inclusion of deformation has any effect on this extrapolation, the correction from the infinite basis was carried out for ${ }^{160} \mathrm{Gd}$ with $\beta_{2}=0.280$ and $\beta_{4}=0.065$ by: 1 ) using the curve deduced above, and 2) actually carrying out diagonalization with a different number of major shells and then carrying out

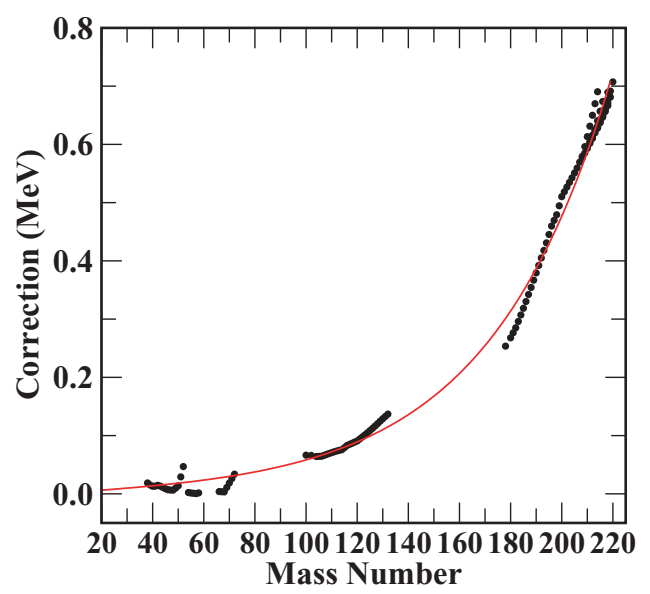

FIG. 9. (Color online) Curve for correcting the ground-state energies obtained by using 15 shells. Note that these corrections are to be subtracted from the calculated ground-state energies. 
an extrapolation as done for Fig. 8. It is observed that the corrections obtained by using these two methods are 0.2067 and 0.2078 , respectively. This indicates that the extrapolation is independent of deformation.

Having established the above equation for correcting quantum mechanical energies, we next proceed to calculate the total binding energy of the 367 spherical nuclei considered in the present work. The strategy is to correct the shell corrections by adding the correction factor deduced above, and then refit the liquid drop parameters. It turns out that the rms deviation of the calculated binding energies thus obtained is $0.633 \mathrm{MeV}$, which is very close to that obtained without correcting the quantum mechanical energies $(0.630 \mathrm{MeV}$; see Sec. IX). Furthermore, the liquid drop parameters differ only marginally from those reported in Sec. IX.

\section{APPENDIX B: GEOMETRY OF DISTANCE FUNCTION}

Consider an arbitrary surface, defined by the relation $r=r_{s}$, where $r_{s}$ is given by Eq. (21) of the text. Let us fix the origin of the coordinate system at the center-of-mass of the object. Let $\vec{r} \equiv(r, \theta, \phi)$ define an arbitrary point in space. This point could be inside or outside the surface. Here, for concreteness, we assume that it is within the volume of the object. Our aim is to find the minimum distance of the point $\vec{r}$ to the surface $r=r_{s}$. To achieve this, construct a vector $\vec{R}_{s}$ from the center-of-mass to the surface. To find the minimum distance, one has to minimize the object $\left|\vec{r}-\vec{R}_{s}\right|$. Denoting the angle between the $\vec{r}$ and $\vec{R}_{S}$ vectors as $\Psi$, we have:

$$
\left|\vec{r}-\vec{R}_{s}\right|=\sqrt{R_{s}^{2}+r^{2}-2 r R_{s} \cos \Psi}
$$

where, from Fig. 10, the cosine of the angle $\Psi$ is given:

$$
\cos \Psi=\cos \theta \cos \theta_{s}+\sin \theta \sin \theta_{s} \cos \left(\phi_{s}-\phi\right) .
$$

The latter result can be proved easily by considering a unit sphere, and a spherical triangle constructed with unit vectors $\hat{r}, \hat{R}_{s}$, and $\hat{z}$. For spherical symmetry, the vectors $\vec{r}$ and $\vec{R}_{s}$ are parallel to each other, and one recovers the usual spherical Woods-Saxon form factor.

\section{APPENDIX C: COULOMB POTENTIAL AND ITS DERIVATIVES}

1. Proof of Eq. (43)

The Coulomb potential for an arbitrary charge distribution is given:

$$
V_{C}(\vec{r})=e^{2} \int \rho\left(\vec{r}^{\prime}\right) \frac{1}{\left|\vec{r}-\vec{r}^{\prime}\right|} d \vec{r}^{\prime} .
$$

Let, for brevity, $\left|\vec{r}-\vec{r}^{\prime}\right|=\mathcal{R}$. Consider,

$$
\vec{\nabla}_{\vec{r}^{\prime}}\left\{\frac{\vec{r}-\vec{r}^{\prime}}{\mathcal{R}}\right\} \text {. }
$$

Here, the symbol $\vec{\nabla}_{\vec{r}^{\prime}}$ means that the differentiation is done with respect to the $r^{\prime}, \theta^{\prime}, \phi^{\prime}$ coordinates. Let us consider the above derivative component-wise. The contribution coming from the first component is

$$
\partial_{x_{1}^{\prime}} \frac{x_{1}-x_{1}^{\prime}}{\mathcal{R}}=\frac{-1}{\mathcal{R}}+\frac{\left(x_{1}-x_{1}^{\prime}\right)^{2}}{\mathcal{R}^{3 / 2}} .
$$

Adding contributions coming from all the three components, one gets

$$
\vec{\nabla}_{\vec{r}^{\prime}} \cdot\left\{\frac{\vec{r}-\vec{r}^{\prime}}{\mathcal{R}}\right\}=\frac{-2}{\mathcal{R}} .
$$

With this, the potential becomes

$$
\begin{aligned}
V_{C}(\vec{r}) & =\frac{-e^{2}}{2} \int \rho\left(\vec{r}^{\prime}\right) \vec{\nabla}_{\vec{r}^{\prime}} \cdot\left\{\frac{\vec{r}-\vec{r}^{\prime}}{\mathcal{R}}\right\} d \vec{r}^{\prime} \\
& =\frac{-e^{2}}{2} \int \rho\left(\vec{r}^{\prime}\right) \vec{\nabla}_{\vec{r}^{\prime}} \cdot \hat{\mathcal{R}} d \vec{r}^{\prime} .
\end{aligned}
$$

Here, the term in the curly brackets has been represented as a unit vector $\hat{\mathcal{R}}$. Using the identity,

$$
\vec{\nabla}_{\vec{r}^{\prime}} \mathcal{R}=-\hat{\mathcal{R}}
$$

one obtains

$$
V_{C}(\vec{r})=\frac{e^{2}}{2} \int \rho\left(\vec{r}^{\prime}\right) \nabla_{\vec{r}^{\prime}}^{2}\left|\vec{r}-\vec{r}^{\prime}\right| d \vec{r}^{\prime},
$$

which, upon integrating by parts and transferring derivatives to density, becomes

$$
V_{C}(\vec{r})=\frac{e^{2}}{2} \int d \vec{r}^{\prime}\left|\vec{r}-\vec{r}^{\prime}\right| \nabla_{\vec{r}^{\prime}}^{2} \rho\left(\vec{r}^{\prime}\right) .
$$

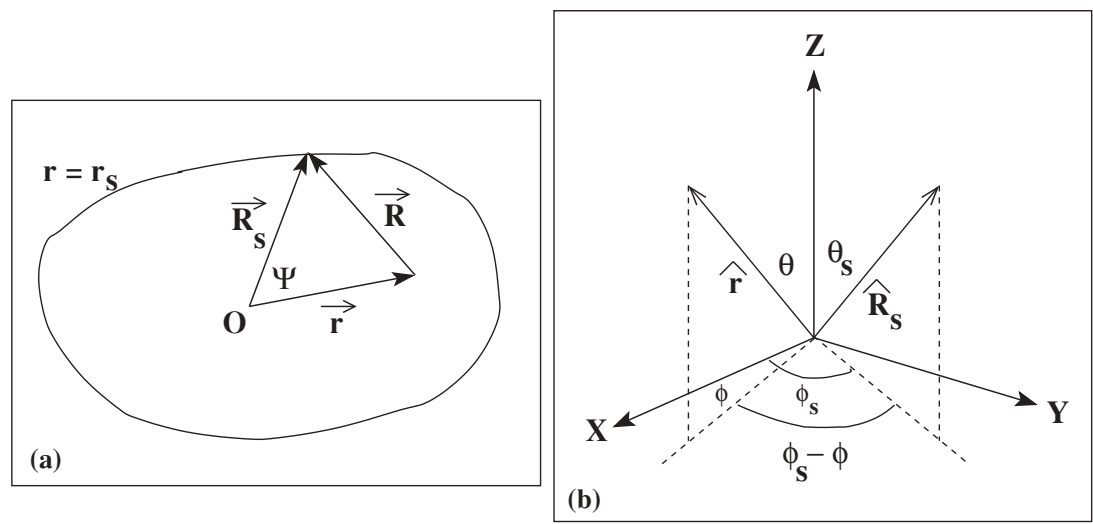

q.e.d. 


\section{Derivatives of Coulomb potential}

The calculation of the higher-order derivatives of the Coulomb potential (third and above), even with the form defined in Eq. (43), turns out to be numerically unstable. For this purpose, we employ the Poisson's equation. According to this, the Laplacian of Coulomb potential is proportional to the charge density:

$$
\nabla^{2} V_{C}(\vec{r})=-4 \pi e^{2} \rho(\vec{r})
$$

The Laplacian of $\nabla^{2} V_{C}(\vec{r})$ is simple to compute, for all one needs to calculate there are the derivatives of density (assumed to be of Woods-Saxon form).

Thus, it is desirable to generate the required higher-order derivatives of the Coulomb potential [see expression Eq. (42) in the text] from Poisson's equation. For this purpose, we evaluate the commutators:

$$
\begin{gathered}
{\left[\nabla^{2} \frac{\partial}{\partial r}, \frac{\partial}{\partial r} \nabla^{2}\right] V_{C}(\vec{r}), \quad\left[\nabla^{2} \frac{1}{r} \frac{\partial}{\partial \theta}, \frac{1}{r} \frac{\partial}{\partial \theta} \nabla^{2}\right] V_{C}(\vec{r}),} \\
{\left[\nabla^{2} \frac{\csc \theta}{r} \frac{\partial}{\partial \theta}, \frac{\csc \theta}{r} \frac{\partial}{\partial \theta} \nabla^{2}\right] V_{C}(\vec{r}) .}
\end{gathered}
$$

The results are

$$
\begin{aligned}
& \nabla^{2} \frac{\partial}{\partial r} V_{C}=\frac{\partial}{\partial r} \nabla^{2} V_{C}+\frac{2}{r}\left[\nabla^{2} V_{C}\right. \\
& \left.-\frac{1}{r} \frac{\partial}{\partial r} V_{C}-\frac{\partial^{2}}{\partial r^{2}} V_{C}\right] \\
& \nabla^{2}\left(\frac{1}{r} \frac{\partial}{\partial \theta} V_{C}\right)=\frac{1}{r} \frac{\partial}{\partial \theta} \nabla^{2} V_{C}+\frac{\csc ^{2} \theta}{r^{3}}\left[\frac{\partial}{\partial \theta} V_{C}\right. \\
& \left.+2 \cot \theta \frac{\partial^{2}}{\partial \phi^{2}} V_{C}\right]-\frac{2}{r^{2}} \frac{\partial^{2}}{\partial \theta \partial r} V_{C} \\
& \nabla^{2}\left(\frac{\csc \theta}{r} \frac{\partial}{\partial \phi} V_{C}\right)=\frac{\csc \theta}{r} \frac{\partial}{\partial \phi} \nabla^{2} V_{C}+\frac{\csc ^{3} \theta}{r^{3}} \frac{\partial}{\partial \phi} V_{C} \\
& -\frac{2 \csc \theta}{r^{2}}\left[\frac{\cot \theta}{r} \frac{\partial^{2}}{\partial \theta \partial \phi} V_{C}+\frac{\partial^{2}}{\partial \theta \partial r} V_{C}\right] \text {. }
\end{aligned}
$$

With these expressions, the required higher-order derivatives of the Coulomb potential can be generated. These are then used to evaluate the fourth-order WK energy, as we have described in Sec. IV.
[1] D. Seweryniak and T. L. Khoo (editors), in AIP Conference Proceedings, Vol. 764, Argonne, 2004 (Springer, New York, 2005).

[2] C. J. Gross, W. Nazarewicz, and K. P. Rykaczewski (editors) in Proceedings of the Conference ENAM'O4 on Exotic Nuclei and Atomic Masses, Pine Mountain, 2004, edited by C. J. Gross, W. Nazarewicz, and K. P. Rykaczewski, Eur. Phys. J. A 25, s01 (2005); in Proceedings of the Conference ENAM'08 on Exotic Nuclei and Atomic Masses, Ryn, Poland, 2008 (Eur. Phys. J. A., in press).

[3] D. Lunney, J. M. Pearson, and C. Thibault, Rev. Mod. Phys. 75, 1021 (2003).

[4] K. Blaum, Phys. Rep. 425, 1 (2006).

[5] Yu. Ts. Oganessian, Joint Institute for Nuclear Research Reprint E7-2002-64.

[6] Yu. Ts. Oganessian et al., Nucl. Instrum. Methods B 204, 606 (2003).

[7] V. S. Kolhinen et al., Nucl. Instrum. Methods A 528, 776 (2004).

[8] S. Goriely, M. Samyn, P. H. Heenen, J. M. Pearson, and F. Tondeur, Phys. Rev. C 66, 024326 (2002), and references cited therein.

[9] S. Goriely, N. Chamel, and J. M. Pearson, Phys. Rev. Lett. 102, 152503 (2009).

[10] S. Hilaire and M. Girod, in Proceedings International Conference on Nuclear Data for Science and Technology, 2007 [online doi:10.1051/ndata:07709 ]. See also http://wwwphynu.cea.fr/HFB-Gogny.htm for the resulting "AMEDEE" structure database.

[11] G. A. Lalazissis, S. Raman, and P. Ring, At. Data Nucl. Data Tables 71, 1 (1999).

[12] P. Möller, J. R. Nix, W. D. Myers, and W. J. Swiatecki, At. Data Nucl. Data Tables 59, 185 (1995).

[13] P. Möller, J. R. Nix, and K.-L. Kratz, At. Data Nucl. Data Tables 66, 131 (1997).
[14] K. Pomorski and J. Dudek, Phys. Rev. C 67, 044316 (2003), and references cited therein.

[15] W. D. Myers and W. J. Swiatecki, Nucl. Phys. A 601, 141 (1996).

[16] V. M. Strutinsky, Yad. Fiz. 3, 614 (1966) [Sov. J. Nucl. Phys. 3, 449 (1966)]; Ark. Fiz. 36, 629 (1966); Nucl. Phys. A 95, 420 (1967).

[17] G. G. Bunatian, V. M. Kolomietz, and V. M. Strutinsky, Nucl. Phys. A 188, 225 (1972).

[18] E. Wigner, Phys. Rev. 40, 749 (1932).

[19] J. G. Kirkwood, Phys. Rev. 44, 33 (1933).

[20] B. K. Jennings, R. K. Bhaduri, and M. Brack, Nucl. Phys. A 253, 29 (1975).

[21] P. Ring and P. Schuck, The Nuclear Many-Body Problem (Springer-Verlag, Berlin, 1980).

[22] M. Brack, C. Guet, and H.-B. Håkansson, Phys. Rep. 123, 275 (1985).

[23] M. Brack and R. K. Bhaduri, Semi-Classical Physics (AddisonWesley Publishing, Reading, 1997).

[24] M. Centelles, P. Leboeuf, A. G. Monastra, J. Roccia, P. Schuck, and X. Vinas, Phys. Rev. C 74, 034332 (2006), and references cited therein.

[25] M. Centelles, P. Schuck, and X. Viñas, Ann. Phys. 322, 363 (2007).

[26] W. Nazarewicz, T. R. Werner, and J. Dobaczewski, Phys. Rev. C 50, 2860 (1994).

[27] S. Shlomo, Nucl. Phys. A 539, 17 (1991).

[28] T. Vertse, A. T. Kruppa, R. J. Liotta, W. Nazarewicz, N. Sandulescu, and T. R. Werner, Phys. Rev. C 57, 3089 (1998).

[29] H. J. Lipkin, Ann. Phys. (NY) 9, 272 (1960).

[30] Y. Nogami, Phys. Rev. 134, B313 (1964).

[31] H. C. Pradhan, Y. Nogami, and J. Law, Nucl. Phys. A 201, 357 (1973).

[32] F. R. Xu, R. Wyss, and P. M. Walker, Phys. Rev. C 60, 051301(R) (1999). 
[33] B. K. Jennings, R. K. Bhaduri, and M. Brack, Phys. Rev. Lett. 34, 228 (1975).

[34] P. Schuck and X. Viñas, Phys. Lett. B 302, 1 (1993).

[35] M. Centelles et al., Ann. Phys. 266, 207 (1998).

[36] B. Grammaticos and A. Voros, Ann. Phys. 123, 359 (1979).

[37] B. Grammaticos and A. Voros, Ann. Phys. 129, 153 (1980).

[38] M. Centelles et al., Nucl. Phys. A 510, 397 (1990).

[39] R. D. Woods and D. S. Saxon, Phys. Rev. 95, 577 (1954).

[40] J. Dudek et al., J. Phys. G: Nucl. Phys. 5, 1359 (1979).

[41] J. Dudek, Z. Szymański, and T. Werner, Phys. Rev. C 23, 920 (1981).

[42] W. Nazarewicz et al., Nucl. Phys. A 435, 397 (1985).

[43] S. Cwiok et al., Comput. Phys. Commun. 46, 379 (1987).

[44] R. Wyss et al., Nucl. Phys. A 511, 324 (1991).

[45] J. Damgaard et al., Nucl. Phys. A 135, 432 (1969).

[46] J. Bennewicz and P. K. Huag, Z. Phys. 212, 212 (1968).

[47] R. Wyss (unpublished).

[48] W. Satula and R. Wyss, Rep. Prog. Phys. 68, 131 (2005), and references cited therein

[49] A. S. Tyapin, Yaz. Fiz. 11, 98 (1970) [Sov. J. Nucl. Phys. 11, 53 (1970)]; Yaz. Fiz. 14, 88 (1972) [Sov. J. Nucl. Phys. 14, 50 (1972)].

[50] D. H. E. Gross, Phys. Lett. B 42, 41 (1972).
[51] A. Sobiczewski et al., Nucl. Phys. A 289, 346 (1977).

[52] B. Mohammed-Azizi and D. E. Medjadi, Phys. Rev. C 74, 054302 (2006).

[53] M. Bolsterli et al., Phys. Rev. C 5, 1050 (1972), and references cited therein.

[54] See supplementary material at http://link.aps.org/supplemental/ 10.1103/PhysRevC.81.044321 for the table. The table can also be found at http://www.ecm.ub.es/ xavier/be_WK.dat. Notice that we take binding energies as negative quantities.

[55] J. Terasaki, J. Engel, and G. F. Bertsch, AIP Conf. Proc. 1128, 48 (2009).

[56] A. H. Wapstra, G. Audi, and C. Thibault, Nucl. Phys. A 729, 129 (2003).

[57] D. W. Marquardt, J. Soc. Ind. Appl. Math. 11, 431 (1963).

[58] W. H. Press, S. A. Teukolsky, W. H. Vetterling, and B. P. Flannery, Numerical Recipes in FORTRAN, 2nd ed. (Cambridge University Press, Cambridge, 1992), p. 678.

[59] E. Rost, Phys. Lett. B 26, 184 (1968).

[60] Y. K. Gambhir and S. H. Patil, Z. Phys. A 321, 161 (1985); A. Bhagwat, Y. K. Gambhir, and S. H. Patil, Eur. Phys. J. A 8, 511 (2000).

[61] T. A. Johansson, Nucl. Phys. A 183, 33 (1971). 